\title{
Magnetic structure determination of high-moment rare-earth-based laminates
}

\author{
D. Potashnikov $\odot,{ }^{1,2,{ }^{*}}$ E. N. Caspi, ${ }^{3}$ A. Pesach, ${ }^{3}$ Q. Tao, ${ }^{4}$ J. Rosen,,${ }^{4}$ D. Sheptyakov,${ }^{5}$ H. A. Evans, ${ }^{6}$ C. Ritter $\odot,{ }^{7}$ Z. Salman $\odot,{ }^{8}$ \\ P. Bonfa, ${ }^{9}$ T. Ouisse,${ }^{10}$ M. Barbier, ${ }^{10}$ O. Rivin, ${ }^{3}$ and A. Keren ${ }^{1}$ \\ ${ }^{1}$ Faculty of Physics, Technion - Israeli Institute of Technology, Haifa 32000, Israel \\ ${ }^{2}$ Israel Atomic Energy Commission, P.O. Box 7061, Tel-Aviv 61070, Israel \\ ${ }^{3}$ Department of Physics, Nuclear Research Centre-Negev, P.O. Box 9001, Beer Sheva 84190, Israel \\ ${ }^{4}$ Materials Design, Department of Physics, Chemistry, and Biology (IFM), Linköping University, SE-581 83 Linköping, Sweden \\ ${ }^{5}$ Laboratory for Neutron Scattering and Imaging, Paul Scherrer Institute, CH-5232 Villigen PSI, Switzerland \\ ${ }^{6}$ Center for Neutron Research, National Institute of Standards and Technology, Gaithersburg, Maryland 20899, USA \\ ${ }^{7}$ Institut Laue-Langevin, 71 Avenue des Martyrs, 38042 Grenoble, France \\ ${ }^{8}$ Laboratory for Muon Spin Spectroscopy, Paul Scherrer Institute, CH-5232 Villigen PSI, Switzerland \\ ${ }^{9}$ Department of Mathematical, Physical and Computer Sciences, University of Parma, 43124 Parma, Italy \\ ${ }^{10}$ Université Grenoble Alpes, CNRS, Grenoble INP, LMGP, F-38000 Grenoble, France
}

(Received 21 September 2021; accepted 8 November 2021; published 29 November 2021)

\begin{abstract}
We report muon spin rotation $(\mu \mathrm{SR})$ and neutron diffraction on the rare-earth-based magnets $\left(\mathrm{Mo}_{2 / 3} \mathrm{RE}_{1 / 3}\right)_{2} \mathrm{AlC}$, also predicted as parent materials for two-dimensional (2D) derivatives, where the rare earth $(\mathrm{RE})=\mathrm{Nd}$, Gd (only $\mu \mathrm{SR})$, Tb, Dy, Ho, and Er. By crossing information between the two techniques, we determine the magnetic moment $(m)$, structure, and dynamic properties of all compounds. We find that only for $\mathrm{RE}=\mathrm{Nd}$ and $\mathrm{Gd}$ the moments are frozen on a microsecond timescale. Out of these two, the most promising compound for a potential 2D high $m$ magnet is the Gd variant, since the parent crystals are pristine with $m=6.5 \pm 0.5 \mu_{\mathrm{B}}$, Néel temperature of $29 \pm 1 \mathrm{~K}$, and the magnetic anisotropy between out-of- and in-plane coupling is smaller than $10^{-8}$. This result suggests that magnetic ordering in the Gd variant is dominated by in-plane magnetic interactions and should therefore remain stable when exfoliated into 2D sheets.
\end{abstract}

DOI: 10.1103/PhysRevB.104.174440

\section{INTRODUCTION}

Two-dimensional (2D) heterostructures based on van der Waals layers require a variety of building blocks including, optionally, magnetic sheets. Up to date, only a handful of transition-metal-based 2D magnets have been found [1-4], and the magnetic moment $m$ of their parent compounds is limited to $4 \mu_{\mathrm{B}}$. Naturally, there is a need for more building blocks. The recently discovered series of crystalline, rareearth (RE) based compounds [5], $\left(\mathrm{Mo}_{2 / 3} \mathrm{RE}_{1 / 3}\right)_{2} \mathrm{AlC} i$-MAX phases, may provide a range of $2 \mathrm{D}$ derivatives to the heterostructure toolbox. Selected $i$-MAX phases, of the general formula $\left(M_{2 / 3}^{\prime} M_{1 / 3}^{\prime \prime}\right)_{2} \mathrm{AlC}$, have previously been shown to realize 2D materials by chemical exfoliation [6]. Importantly, through the concept of "targeted etching," the 2D sheets can be tailored to retain one or both of the $M$ elements [6], suggesting RE-based 2D materials from the three-dimensional (3D) atomic laminates investigated herein. Furthermore, theoretical predictions [7] and preliminary experimental results (not shown) suggest derivation of magnetic $2 \mathrm{D}$ sheets also from mechanical exfoliation [8].

However, not much is known on the magnetic structure of the parent compounds, let alone on the potential 2D sheets. Temperature- and field-dependent magnetization and heat ca-

*sdannyp@campus.technion.ac.il pacity measurements [5] on $\left(\mathrm{Mo}_{2 / 3} \mathrm{RE}_{1 / 3}\right)_{2} \mathrm{AlC}$ have shown that most compounds are magnetically ordered with transition temperatures ranging from $3.6 \mathrm{~K}$ for $\mathrm{RE}=\mathrm{Er}$ up to $28 \mathrm{~K}$ for $\mathrm{RE}=\mathrm{Tb}$. Curie-Weiss plots of inverse susceptibility versus temperature gave negative Weiss temperatures, suggesting that the magnetic coupling is antiferromagnetic (AFM). Neutron powder diffraction (NPD) on RE $=\mathrm{Tb}$ and Er found that both compounds order as spin density waves (SDWs), confirming the AFM assumption at least in these two compounds.

In this work the magnetic ground states of bulk powder $\left(\mathrm{Mo}_{2 / 3} \mathrm{RE}_{1 / 3}\right)_{2} \mathrm{AlC}$ with $\mathrm{RE}=\mathrm{Nd}, \mathrm{Gd}, \mathrm{Tb}, \mathrm{Dy}, \mathrm{Ho}$, and $\mathrm{Er}$ are explored using muon spin rotation $(\mu \mathrm{SR})$. The $\mathrm{Gd}$ variant is also explored in a single-crystal form, as a function of initial muon polarization direction. In addition, the series of powders is examined with NPD, excluding $\mathrm{RE}=\mathrm{Gd}$, since it is a strong neutron absorber. From NPD, it is found that, like $\mathrm{RE}=\mathrm{Tb}$ and $\mathrm{Er}$ in previous work [5], all compounds except $\mathrm{RE}=$ Gd develop a SDW magnetic ordering with their propagation vector directed along the unique axis of the monoclinic unit cell. Using density functional theory (DFT) based muon site calculations, symmetry analysis, and information from the $\mathrm{NPD}$ data on $\mathrm{RE}=\mathrm{Nd}, \mathrm{Tb}, \mathrm{Ho}$, and $\mathrm{Er}$, the magnetic structure of $\mathrm{RE}=\mathrm{Gd}$ is also determined to be a simple AFM with an extremely small anisotropy ratio between in- and out-of-plane coupling. Moreover, only $\mathrm{RE}=\mathrm{Nd}$ and $\mathrm{Gd}$ exhibit long-range static magnetic order within the $\mu \mathrm{SR}$ time window (10 $\mu \mathrm{s})$, while for other REs, the magnetic order is quasistatic down to 
a temperature of $1.5 \mathrm{~K}$. Quasistatic refers to fluctuations that are on a timescale longer than the typical neutron scattering time window of $10^{-12} \mathrm{~s}$ but shorter than or equal to the $\mu \mathrm{SR}$ time window.

\section{EXPERIMENTAL DETAILS}

\section{A. Sample preparation and characterization}

Polycrystalline samples are synthesized by pressureless sintering the following elemental powders: graphite (99.999\%), Mo (99.99\%) from Sigma-Aldrich, Al (99.8\%) from Alfa Aesar, and RE (99.9\%) from Stanford Advanced Materials. Stoichiometric amounts of the elemental powders are manually mixed in an agate mortar and then placed in an alumina crucible that is in turn inserted in an alumina tube furnace through which $5 \mathrm{sccm}$ of $\mathrm{Ar}$ is flowing. The furnace is then heated, at $5{ }^{\circ} \mathrm{C}$ per minute, up to $1500{ }^{\circ} \mathrm{C}$ and held at $1500{ }^{\circ} \mathrm{C}$ for $10 \mathrm{~h}$ before furnace cooling to room temperature. The loosely sintered powders are crushed into a fine powder that is directly used for further analysis.

$\left(\mathrm{Mo}_{2 / 3} \mathrm{Gd}_{1 / 3}\right)_{2} \mathrm{AlC}$ single crystals are grown using a flux growth technique in an induction-heated growth reactor and a sealed graphite crucible. Sealing is mandatory for avoiding $\mathrm{RE}$ and Al evaporation, which implies reliance on spontaneous nucleation inside the flux in order to produce the crystals. Initial molar ratios $x$ before carbon incorporation are $x_{\mathrm{Mo}}=0.1, x_{\mathrm{Gd}}=0.4$, and $x_{\mathrm{Al}}=0.5$. After applying a maximum temperature of $1800{ }^{\circ} \mathrm{C}$ for $1 \mathrm{~h}$, required for melting the Mo element, the growth reactor is slowly cooled to $1000{ }^{\circ} \mathrm{C}$ in 7 days. The source of $\mathrm{C}$ is the graphite crucible walls, and typical $\mathrm{C}$ concentrations in the flux may range from $x_{\mathrm{C}}=$ $0.2-0.4$, as estimated from weighting the crucible before and after growth. After growth, single crystals are extracted from the solidified flux by oxidizing the latter inside a chamber equipped with an air flux bubbling in water. The maximum lateral crystal size rarely exceeded $1 \mathrm{~mm}$, and each growth resulted in hundreds of small single crystals with a wide distribution in size. Single crystals with a lateral size larger than $100 \mu \mathrm{m}$ were selected. In addition to crystals grown in those optimal conditions, crystals produced with different growth conditions (cooling during 2 days and slightly different flux compositions) were also collected.

Room-temperature powder $\mathrm{X}$-ray diffraction (XRD) patterns of all compounds were recorded using the $X^{\prime}$ Pert Pro diffractometer using $\mathrm{Cu} \mathrm{K} \alpha$ radiation. A step size of $0.017^{\circ}$ and dwell time of $20 \mathrm{~s}$ for each step was used. The diffraction profiles were analyzed using Rietveld refinement as implemented within the FULLPROF suite [9].

\section{B. Neutron powder diffraction}

NPD of compounds with $\mathrm{RE}=\mathrm{Nd}$ and $\mathrm{Tb}$ was performed at the D20 neutron diffractometer [10] located at the Institut Laue-Langevin (ILL), France. A wavelength of $2.4166 \AA$ was obtained using a pyrolytic graphite monochromator $(002$ reflection) and no additional collimation. The samples were loaded into a vanadium holder of $8 \mathrm{~mm}$ diameter and cooled using a standard ILL Orange cryostat [11].

High-resolution NPD of $\mathrm{RE}=\mathrm{Er}$ is performed at the high-resolution powder diffractometer for thermal neutrons
(HRPT) [12] located at the Paul Scherrer Institute, Switzerland. An incident wavelength of $1.886 \AA$ was obtained using a focusing $\mathrm{Ge}(511)$ monochromator with no additional collimation. The sample was loaded into a vanadium holder of $12 \mathrm{~mm}$ diameter, cooled using a standard ILL Orange cryostat, and measured in the high-intensity mode.

Additional high-resolution NPD measurements were performed on compounds with $\mathrm{RE}=\mathrm{Er}$ and Ho using the BT-1 diffractometer [13] at the National Center for Neutron Research located at the National Institute of Standards and Technology, USA. An incident wavelength of $2.077 \AA$ was obtained using the $\mathrm{Ge}(311)$ monochromator and an in-pile collimation of 60'. The samples were loaded into a vanadium holder with a diameter of $9.2 \mathrm{~mm}$. $\mathrm{A}^{3} \mathrm{He}$ cryostat [14] was used for the $\mathrm{RE}=\mathrm{Er}$ sample, while the $\mathrm{RE}=$ Ho sample was loaded into a low- $T$ high-power closed cycle refrigerator (CCR) [15].

NPD patterns for all measured compounds were collected at temperatures that range from the available base temperature (BT) up to the magnetic transition temperature and into the paramagnetic (PM) phase. An additional measurement of $\mathrm{RE}=\mathrm{Er}$ was performed at $0.3 \mathrm{~K}$ using the ${ }^{3} \mathrm{He}$ cryostat Rietveld analysis of the data was performed using the FULLPROF [9] suite. Symmetry analysis and the propagation vector search were handled by BASIREPS [16] and SARAH [17], respectively. Visualization of all crystal and magnetic structures in this paper was done using VESTA [18].

\section{Muon spin rotation}

All $\mu$ SR measurements were done at the general-purpose spectrometer [19] (GPS) at the Paul Scherrer Institute, Switzerland. Powder samples of $\left(\mathrm{Mo}_{2 / 3} \mathrm{RE}_{1 / 3}\right)_{2} \mathrm{AlC}$ were pressed into pellets of $10 \mathrm{~mm}$ diameter and $5 \mathrm{~mm}$ thickness, mounted using aluminized Mylar tape and cooled down in a He gas flow cryostat [20]. All samples are measured from a $\mathrm{BT}$ of $1.5 \mathrm{~K}$ up to their respective PM temperatures.

Single crystals of $\left(\mathrm{Mo}_{2 / 3} \mathrm{Gd}_{1 / 3}\right)_{2} \mathrm{AlC}$ with lateral sizes greater than $100 \mu \mathrm{m}$ were selected from each growth and glued on a silver plate sample holder using General Electric (GE) varnish. The crystallites with thickness ranging from 10 to $100 \mu \mathrm{m}$ grew preferentially along their basal planes and therefore the $c^{*}$ axis was perpendicular to their largest surface. The crystals are arranged such that the $c^{*}$ axis is perpendicular to the sample holder plane but are not oriented in the $a-b$ plane. The resulting sample is disk shaped with a diameter of $10 \mathrm{~mm}$ and a thickness of $\approx 3 \mathrm{~mm}$. The single-crystal sample was measured at $1.5 \mathrm{~K}$ for different initial muon polarization directions $\zeta=4.7^{\circ}, 11^{\circ}, 19^{\circ}, 28^{\circ}, 38^{\circ}$, and $53^{\circ}$ using two sets of detectors: up-down and forward-backward. The muon polarization direction was controlled by setting appropriate voltage and current values in the GPS spin rotator [19]. It was then experimentally determined by measuring the sample at $30 \mathrm{~K}$ with an applied transverse field of $30 \mathrm{G}$ and fitting a simple cosine function to the data. The fitted phase in the up-down detectors determines the muon polarization direction. All measurements were recorded in a time window of $3 \mu$ s with a 98-ps time resolution. Temperature-dependent measurements of the single-crystal sample were performed at the same temperatures as the $\mathrm{RE}=\mathrm{Gd}$ powder sample. 


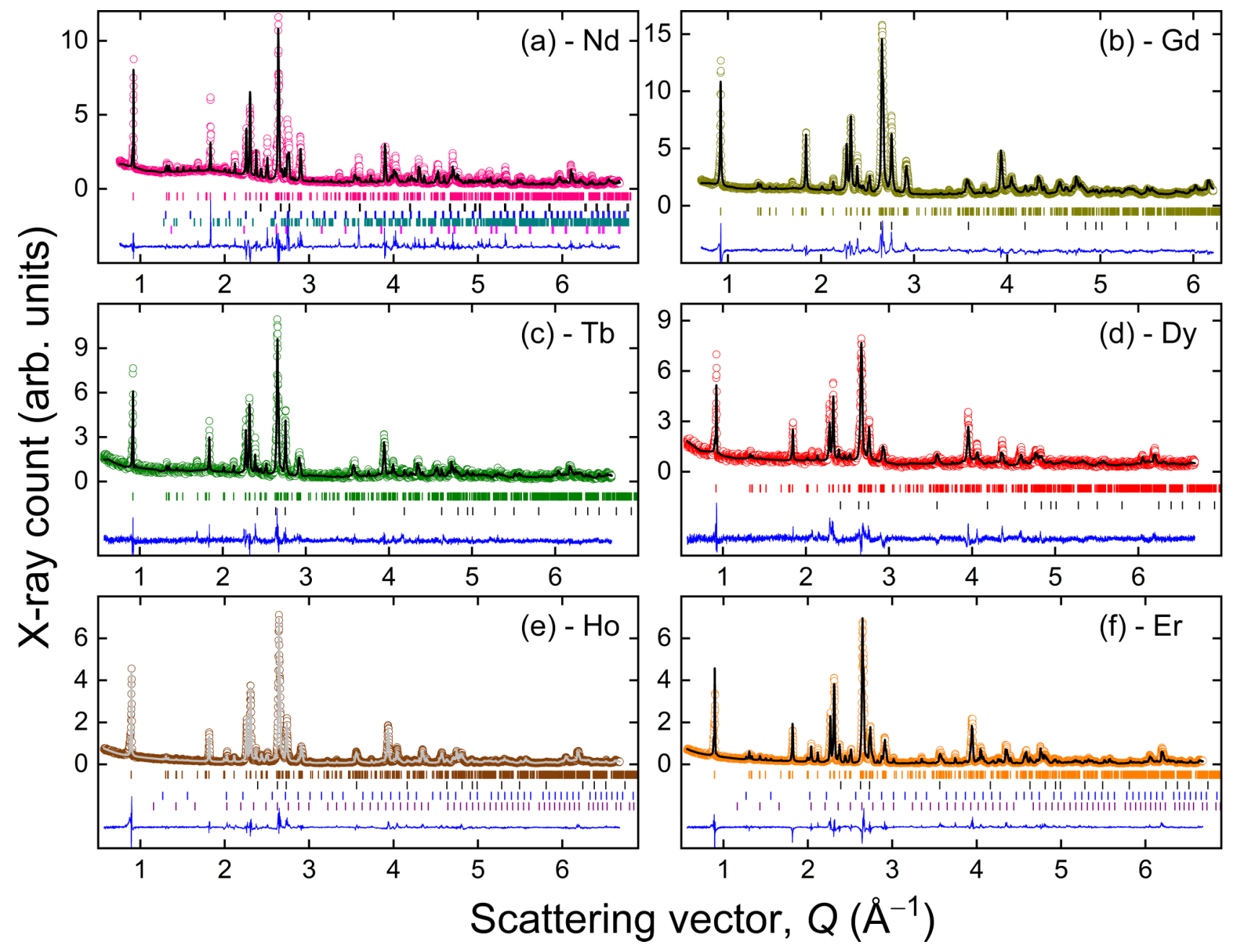

FIG. 1. Observed XRD patterns of $\left(\mathrm{Mo}_{2 / 3} \mathrm{RE}_{1 / 3}\right)_{2} \mathrm{AlC}$ compounds (symbols) obtained for $\mathrm{RE}=$ (a) $\mathrm{Nd}$, (b) $\mathrm{Gd}$, (c) $\mathrm{Tb}$, (d) $\mathrm{Dy}$, (e) Ho, and (f) Er at room temperature, the corresponding Rietveld refined profile (solid line on data), and their difference (solid bottom blue line). Positions of reflections belonging to the main phase, as well as any impurity phases, are denoted as tick marks. The order of tick marks from top to bottom in each plot corresponds to the order of impurities given in Table S1 [25]. Uncertainties of data points (1 $\sigma)$ are represented by their spread around the refined profile.

\section{Density functional theory calculations}

DFT calculations were performed using the QUANTUM ESPRESSO suite of codes [21]. The generalized gradient approximation (GGA) as parameterized by Perdew-BurkeErnzerhof (PBE) [22] was used to describe exchange correlation effects and a non-spin-polarized approach was used. All calculations were performed on a $6 \times 8 \times 4 k$-point grid with a $70 \mathrm{Ry}(950 \mathrm{meV})$ cutoff energy with the wave functions of the $4 f$ electrons treated as core states. The initial atomic positions for all materials were taken from the low-temperature NPD measurements, except $\mathrm{RE}=\mathrm{Gd}$ where the atomic positions were taken from RT XRD measurements. The positive muon was approximated by a hydrogen atom occupying interstitial positions in the lattice. The search for candidate muon sites was performed by splitting the asymmetric unit of the $C 2 / c$ unit cell into a $3 \times 3 \times 3$ grid, with the muon placed in one of the 27 starting positions. All atoms in the unit cell were then relaxed until forces are smaller than $10^{-3} \mathrm{Ry} /$ Bohr radius $(26 \mathrm{meV} / \AA)$ and energy differences smaller than $10^{-4} \mathrm{Ry}(1.4$
meV). The final energy was then used to determine the most likely muon site.

\section{RESULTS}

\section{A. X-ray powder diffraction}

XRD patterns of all samples (Fig. 1) contain reflections consistent with a monoclinic $(C 2 / c)$ unit cell having lattice parameters (LPs) $a, b, c$, and $\beta$ in the ranges 9.48-9.67 $\AA$, 5.49-5.6 $\AA$, $14.01-14.17 \AA$, and 103.48-103.57 , respectively, with the $b$ axis defined as the unique axis. Additional reflections belonging to impurity phases in each sample are identified by systematically testing all phases containing RE, $\mathrm{Mo}, \mathrm{Al}, \mathrm{C}$, and $\mathrm{O}$. The main impurities found are $\mathrm{Mo}_{2} \mathrm{C}$ $(P 63 / m m c), \mathrm{Mo}_{3} \mathrm{Al}_{2} \mathrm{C}\left(P 4_{1} 32\right)$, and $\mathrm{RE}_{2} \mathrm{O}_{3}(I a-3)$. Structural models for the main phase and all impurities are taken from Ref. [5]. The refinement then consists of the main monoclinic $\left(\mathrm{Mo}_{2 / 3} \mathrm{RE}_{1 / 3}\right)_{2} \mathrm{AlC}$ compound, with all identified impurities (Table S1 [25]), with the unit cell parameters and atomic posi- 
TABLE I. LPs $(a, b, c, \beta)$, unit-cell volume $(V)$, mass percent (wt \%), and agreement factor (Rf) [23] obtained from Rietveld refinement of room-temperature XRD. Numbers in parentheses indicate $1 \sigma$ statistical uncertainties of the refinement.

\begin{tabular}{|c|c|c|c|c|c|c|c|}
\hline $\mathrm{Nd}$ & $9.6675(3)$ & $5.5946(2)$ & $14.1700(4)$ & $103.481(2)$ & $745.27(4)$ & $89(1)$ & 17.6 \\
\hline $\mathrm{Tb}$ & $9.5420(4)$ & $5.5221(2)$ & $14.0601(6)$ & $103.541(2)$ & $720.26(5)$ & 91.6(9) & 16.7 \\
\hline Dy & $9.5315(7)$ & $5.5059(4)$ & $14.0413(9)$ & $103.549(4)$ & $716.36(8)$ & $97(1)$ & 18.1 \\
\hline Ho & $9.5075(3)$ & $5.5012(1)$ & $14.0306(4)$ & $103.527(2)$ & $713.48(3)$ & 93.1(8) & 16.5 \\
\hline
\end{tabular}

tions of the $\left(\mathrm{Mo}_{2 / 3} \mathrm{RE}_{1 / 3}\right)_{2} \mathrm{AlC}$ refined. Refinement results of the XRD data are given in Table I.

\section{B. Ground-state neutron powder diffraction}

NPD patterns for all samples at the PM phase [cf. Fig. 2(a)] contain reflections consistent with a monoclinic $(C 2 / c)$ unit cell having LPs $a, b, c$, and $\beta$ in the ranges 9.48-9.67 $\AA, 5.49-$ 5.6 $\AA$, 14.01-14.17 $\AA$, and 103.48-103.57 ${ }^{\circ}$, respectively, as the main phase, in agreement with XRD measurements. For $\mathrm{RE}=$ Dy data and analysis, see Ref. [24]. $\mathrm{RE}=\mathrm{Er}$ data is examined in detail, with data of the magnetically ordered phase shown in Fig. 2(b). Upon cooling, clear additional reflections originating from magnetic diffraction are observed and emphasized by arrows in Fig. 2(b).
Refinement results from XRD (Fig. 1) are used as initial guesses for the paramagnetic NPD pattern [Fig. 2(a)]. Instrumental resolution and wavelength are obtained from measurement of standards and kept fixed. The refined variables are LPs, atomic positions, scale factors, LPs of impurity phases, and instrumental zero shift. Following the refinement of the PM phase, a similar refinement is performed for BT with the additional magnetic phase excluded.

To isolate the magnetic contribution from the crystallographic reflections, the difference between BT and PM temperatures is calculated [Fig. 2(c) and Fig. S1 in the Supplemental Material [25]] and analyzed first. To analyze the difference pattern between BT and PM temperatures, only the magnetic phase is considered. Its scale factor, LPs, and atomic positions are fixed by the BT crystallographic refinement of the crystallographic reflections. Initial analysis
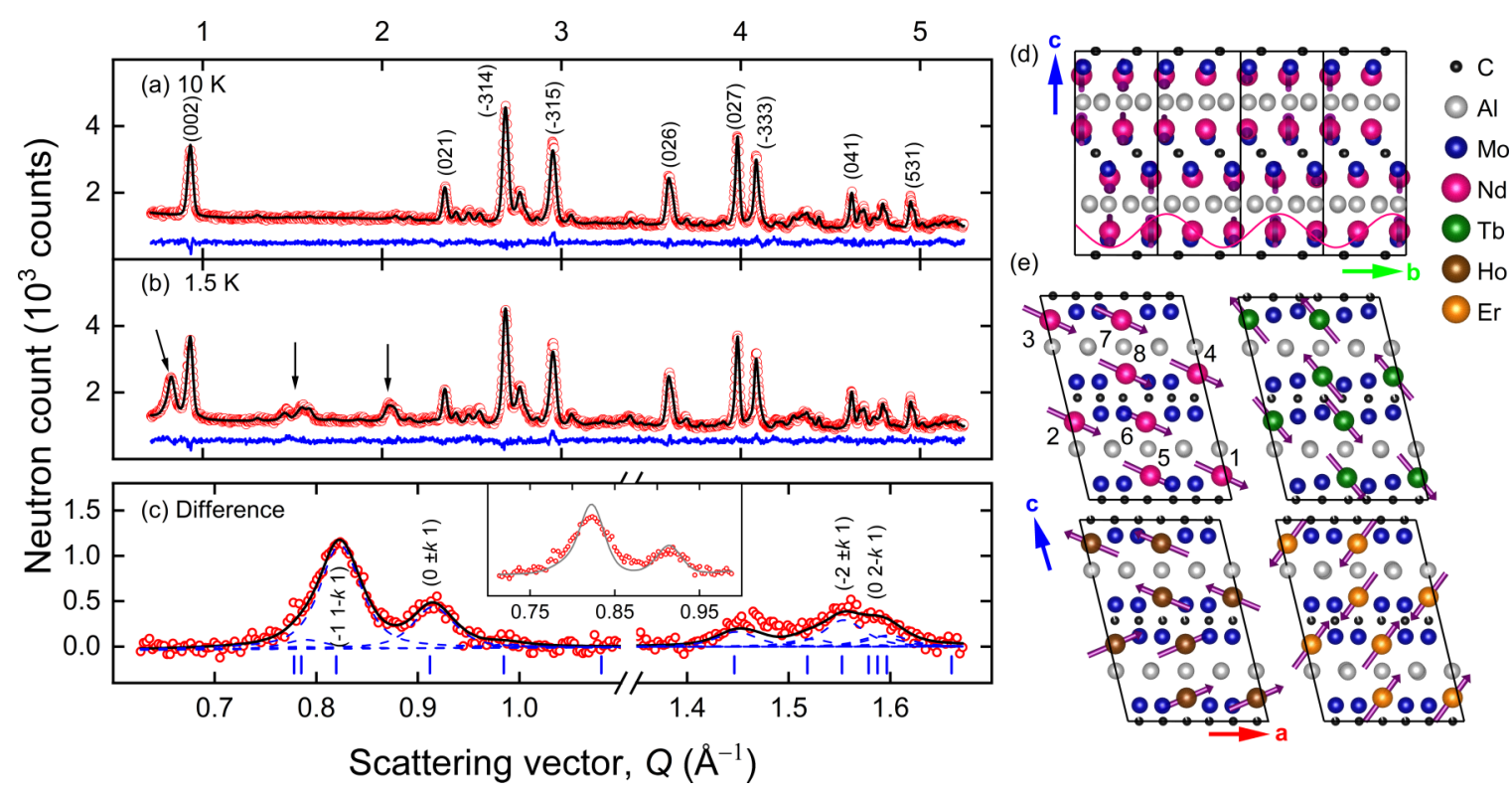

FIG. 2. Observed (symbols) neutron powder diffraction profiles of $\left(\mathrm{Mo}_{2 / 3} \mathrm{Er}_{1 / 3}\right)_{2} \mathrm{AlC}$ collected with the HRPT diffractometer at (a) $10 \mathrm{~K}$, (b) $1.6 \mathrm{~K}$, and (c) their difference. Arrows in (b) show magnetic reflections which appear upon cooling. The calculated profile and its difference from the data are shown as solid black and blue lines, respectively. Contributions from individual magnetic reflections in (c) are shown as dashed lines. Crystallographic and magnetic reflections of the main phase are labeled by their Miller indices and tick marks. Inset in (c) shows the calculated profile without including Lorentzian broadening (see text). Uncertainties of data points $(1 \sigma)$ are represented by their spread around the refined profile. (d) Depiction of the SDW structure for $\left(\mathrm{Mo}_{2 / 3} \mathrm{Nd}_{1 / 3}\right)_{2} \mathrm{AlC}$ in the $b$ - $c$ plane. (e) Unit-cell spin configurations of $\left(\mathrm{Mo}_{2 / 3} \mathrm{RE}_{1 / 3}\right)_{2} \mathrm{AlC}$, where $\mathrm{RE}=\mathrm{Nd}, \mathrm{Tb}, \mathrm{Ho}$, and $\mathrm{Er}$ at base temperature. The spin configuration for $\left(\mathrm{Mo}_{2 / 3} \mathrm{~Tb}_{1 / 3}\right)_{2} \mathrm{AlC}$ shows the commensurate $\mathbf{k}_{1}=(0,0.5,0)$ structure. The modulation in the magnetic moment magnitude acquired from the propagation vector (see text) is removed in this figure, and all magnetic moments are rescaled for clarity. Numbers in (e) indicate order of magnetic atoms according to the international tables for crystallography. 
TABLE II. Refined magnetic structure parameters for $\left(\mathrm{Mo}_{2 / 3} \mathrm{RE}_{1 / 3}\right)_{2} \mathrm{AlC}$ phases. $T_{\mathrm{N}}$ is the Néel temperature, BT is the base temperature at which the measurement is performed, $m$ is the ordered magnetic moment per atom, $\theta$ is the orientation of the magnetic moment relative the $z$ axis (see text), $k$ is the propagation vector component along the $b$ axis, $\xi_{\mathrm{RE}}$ is the magnetic correlation length, and $R_{\text {mag }}$ is the magnetic agreement factor [9]. Numbers in parenthesis indicate $1 \sigma$ statistical uncertainties, while \pm indicates systematic uncertainty. Dash indicates the data falls below the limit of detection.

\begin{tabular}{lcccccc}
\hline \hline $\mathrm{RE}$ & $T_{\mathrm{N}}(\mathrm{K})[5]$ & $\mathrm{BT}(\mathrm{K})$ & $m_{\mathrm{RE}}\left(\mu_{\mathrm{B}} / \mathrm{RE}\right)$ & $\theta_{\mathrm{RE}}(\mathrm{deg})$ & $k_{\mathrm{RE}}$ & $\xi_{\mathrm{RE}}(\mathrm{nm})$ \\
\hline $\mathrm{Nd}$ & 7.6 & 1.5 & $1.76(2)$ & $115(2)$ & $0.730(2)$ & - \\
$\mathrm{Gd}$ & 26 & & $6.6 \pm 0.3$ & $120 \pm 7$ & 0.5 & - \\
$\mathrm{Tb}$ & 20.1 & 3.5 & $5.63(3)$ & $132(2)$ & 0.5 & - \\
& 28 & & $3.1(1)$ & $126(2)$ & $0.636(1)$ & - \\
$\mathrm{Ho}$ & 7.8 & 4 & $8.7(2)$ & $67(1)$ & $0.6717(7)$ & 14.7 \\
$\mathrm{Er}$ & 3.6 & 1.6 & $6.54(6)$ & $36.6(9)$ & $0.6787(3)$ & 29.2 \\
\hline \hline
\end{tabular}

of the difference pattern consists of a propagation vector search with simulated-annealing-based refinement, as implemented in SARAH [17]. To find possible spin configurations within the unit cell, irreducible representations for the little group of the $k$ vector are calculated using BASIREPS [16]. More details on the calculation and basis vectors is given in the Supplemental Material [25]. For each magnetic representation, up to six basis vector coefficients can be refined. To find the best fitting magnetic configuration, a systematic refinement of all possible combinations of basis vectors is performed. The best choice is selected by requiring a minimum magnetic agreement factor $\left(R_{\mathrm{mag}}\right)$ [23] while also searching for the solution described by the least number of basis vector coefficients with physical magnetic moments. The final parameters for the BT magnetic structure are obtained by adding the magnetic contribution to the crystallographic profile and refining both magnetic and crystal structures simultaneously. In the final refinement, scale factors and LPs of all phases are refined, as well as atomic positions of the main phase.

Refinement of the BT magnetic structures reveals that the magnetic ground states of compounds with $\mathrm{RE}=\mathrm{Nd}, \mathrm{Tb}, \mathrm{Ho}$, and $\mathrm{Er}$ are SDWs with propagation vectors $\mathbf{k}_{\mathrm{RE}}$ parallel to the crystallographic $b$ axis [Fig. 2(d)]. The magnetic moments of the $\mathrm{RE}$ atoms are aligned perpendicular to $\mathbf{k}_{\mathrm{RE}}$ with magnetic directions varying with RE [Fig. 2(e)]. Three different magnetic configurations in the unit cell are identified and labeled using Bertaut notation [26] as $\mathrm{F}_{\mathrm{x}} \mathrm{F}_{\mathrm{z}}$ [Fig. 2(e), $\mathrm{RE}=\mathrm{Nd}$ ], $\mathrm{C}_{\mathrm{x}} \mathrm{C}_{\mathrm{z}}$ [Fig. 2(e), Tb, Er], and $\mathrm{C}_{\mathrm{x}} \mathrm{F}_{\mathrm{z}}$ [Fig. 2(e), Ho]. All three configurations are obtained from the $\Gamma_{2}$ representation (see Supplemental Material [25]), where the magnetic moments of RE atoms 1 and 2 [Fig. 2(e)] are parallel and oriented in the $a-c$ plane.

Attempting to refine the BT profile of $\mathrm{RE}=\mathrm{Er}$ and $\mathrm{Ho}$ using the instrumental resolution resulted in calculated reflections which are narrower than the observed reflections [Fig. 2(c), inset]. To obtain a better fitting peak shape, an additional Lorentzian contribution is added to the magnetic reflections (FULLPROF $Y$ parameter [9]). The magnetic correlation length is then estimated using the Scherrer formula $\xi=$ $0.8 \lambda / Y$ [27]. For $\mathrm{RE}=\mathrm{Nd}$ and $\mathrm{Tb}$, no additional broadening is observed within the limits of the instrumental resolution, and thus $\xi$ cannot be determined, and assumed to match the crystallographic correlation length. $\mathrm{RE}=\mathrm{Nd}$ shows the weakest reflections and has the worst agreement factor (Table II).
To limit the number of refined parameters, the magnetic moments are constrained to lie in the $a-c$ plane in accordance with the other $\left(\mathrm{Mo}_{2 / 3} \mathrm{RE}_{1 / 3}\right)_{2} \mathrm{AlC}$ phases.

Refinement of the BT measurement of $\mathrm{RE}=\mathrm{Tb}$ requires a magnetic structure comprised of two propagation vectors (Table II). The basis vector coefficient refinement is done for each propagation vector separately. The best fitting combination of basis vectors for both propagation vectors gives magnetic moments which are oriented in the $a-c$ plane. The final BT magnetic structures are shown in Figs. 2(d) and 2(e), and their properties are summarized in Table II.

\section{Ground-state muon spin rotation}

The asymmetry in the decay positrons at BT and zero applied field of different $\left(\mathrm{Mo}_{2 / 3} \mathrm{RE}_{1 / 3}\right)_{2} \mathrm{AlC}$ samples is plotted in Figs. 3(a) and 3(b). Three distinct behaviors are observed:

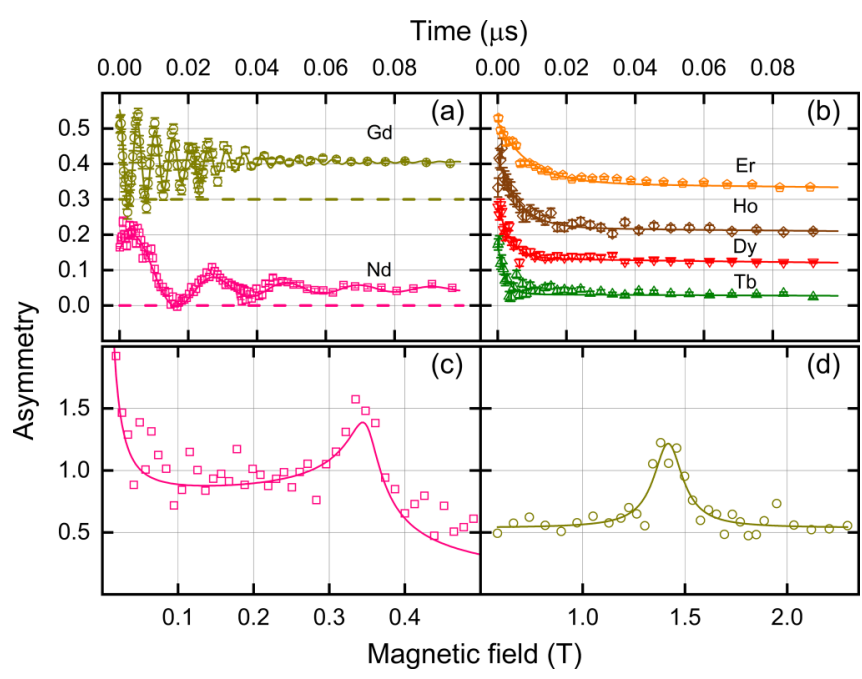

FIG. 3. Observed BT asymmetry as a function of time from muon implantation (symbols) of (a) $\mathrm{RE}=\mathrm{Nd}$ and $\mathrm{RE}=\mathrm{Gd}$, (b) $\mathrm{RE}=\mathrm{Tb}, \mathrm{Dy}, \mathrm{Ho}$, and Er. Data in (a) and (b) is shifted for clarity (for $\mathrm{RE}=\mathrm{Gd}$ by 0.3 and for $\mathrm{RE}=\mathrm{Dy}$, Ho and $\mathrm{Er}$ by $0.1,0.2$, and 0.3 , respectively). Error bars represent $1 \sigma$ statistical uncertainty on the data points. Dashed lines in (a) indicate zero asymmetry for each data set. Magnetic field distribution for (c) $\mathrm{RE}=\mathrm{Nd}$ and (d) $\mathrm{RE}=$ Gd obtained by Fourier transforming the data in (a). Solid lines show fits to the data. 
TABLE III. Fit results for BT asymmetry of different $\left(\mathrm{Mo}_{2 / 3} \mathrm{RE}_{1 / 3}\right)_{2} \mathrm{AlC}$ compounds. $A_{F / S}$ is the fast/slow relaxing asymmetry amplitude, $B$ is the static magnetic field, and $\lambda_{F / S}$ is the relaxation rate of the fast/slow component. Numbers in parenthesis indicate $1 \sigma$ statistical uncertainties. Dash indicates irrelevant data.

\begin{tabular}{lcccll}
\hline \hline $\mathrm{RE}$ & Asymmetry function & $A_{F} / A_{S}$ & $B(\mathrm{~T})$ & $\lambda_{F}\left(\mu \mathrm{s}^{-1}\right)$ & $\lambda_{S}\left(\mu \mathrm{s}^{-1}\right)$ \\
\hline $\mathrm{Nd}$ & $A_{1}$ & $3.29(2)$ & $0.350(2)$ & $14(1)$ & $0.42(1)$ \\
$\mathrm{Gd}$ & $A_{2}$ & $1.93(1)$ & $1.426(3)$ & $49(2)$ & $0.053(5)$ \\
$\mathrm{Tb}$ & $A_{3}$ & $4.43(5)$ & - & $440(50)$ & $1.40(7)$ \\
$\mathrm{Dy}$ & & $4.53(7)$ & - & $270(20)$ & $4.7(3)$ \\
$\mathrm{Ho}$ & & $9.2(2)$ & - & $160(15)$ & $7.5(8)$ \\
$\mathrm{Er}$ & & $3.68(3)$ & - & $113(4)$ & $3.2(1)$ \\
\hline \hline
\end{tabular}

(a) $\mathrm{RE}=\mathrm{Nd}$ shows oscillations which closely resemble a Bessel function. (b) Similarly, RE $=$ Gd shows oscillations which are well described by a cosine function. (c) In contrast, other REs show an exponential decay characterized by a fast component $(t<0.1 \mu \mathrm{s})$ and a slow component. For all compounds, the asymmetry signal decays to zero at long times $(\approx 3 \mu \mathrm{s}$, not shown) and does not show the characteristic 1/3 component of powders, had there been an internal static magnetic field parallel to the muon spin [28]. Fourier transforms of the observed asymmetry in $\mathrm{RE}=\mathrm{Nd}$ and $\mathrm{Gd}$ [Figs. 3(c) and 3(d), respectively] show one dominant field in each sample, namely, $0.35 \mathrm{~T}$ and $1.4 \mathrm{~T}$ for $\mathrm{RE}=\mathrm{Nd}$ and $\mathrm{Gd}$, respectively. The broadened field spectrum of $\mathrm{RE}=$ $\mathrm{Nd}$ is characteristic of an incommensurate magnetic structure
[29], while a single well-defined peak in the $\mathrm{RE}=\mathrm{Gd}$ field spectrum corresponds to a simple commensurate structure [ferromagnet (FM) or AFM]. Following these observations, the asymmetry functions used as fits to the data are

$$
\begin{gathered}
A_{1}(t)=A_{F} J_{0}\left(\gamma_{\mu} B t+\phi\right) e^{-\lambda_{F} t}+A_{S} e^{-\lambda_{S} t} \\
A_{2}(t)=A_{F} \cos \left(\gamma_{\mu} B t+\phi\right) e^{-\lambda_{F} t}+A_{S} e^{-\lambda_{S} t} \\
A_{3}(t)=A_{F} e^{-\lambda_{F} t}+A_{S} e^{-\lambda_{S} t}
\end{gathered}
$$

where $F$ and $S$ stand for fast and slow relaxing signals, respectively, $A_{F / S}$ are the amplitudes of each term, $\lambda_{F / S}$ are the relaxation rates, and $J_{0}$ is a zeroth-order Bessel function. $A_{1}$ is used for $\mathrm{RE}=\mathrm{Nd}, A_{2}$ for $\mathrm{RE}=\mathrm{Gd}$, and $A_{3}$ is used for the rest. The final fit parameters for each RE (Table III) are obtained after simultaneous fitting to all temperature-dependent spectra (see Sec. III E).

In order to convert the magnetic field distribution experienced by the muon into the magnetic moment distribution, the muon site has to be determined [30]. This is done in three steps. First, the stable muon site in $\mathrm{RE}=\mathrm{Nd}$ is determined using an adaptation of DFT [31], which allows relaxation of the lattice in the presence of the muon. Second, the expected magnetic moment in $\mathrm{RE}=\mathrm{Nd}$ is calculated using $\mu \mathrm{SR}$ results and compared to the NPD results to verify the validity of the DFT approach. Finally, the same procedure is applied to RE = $\mathrm{Gd}$, where the magnetic structure is not determined by NPD. (a)

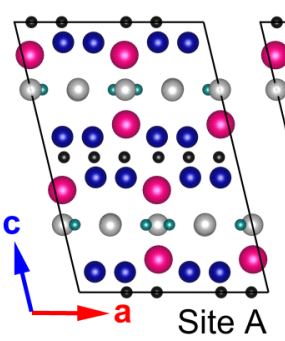

(c)

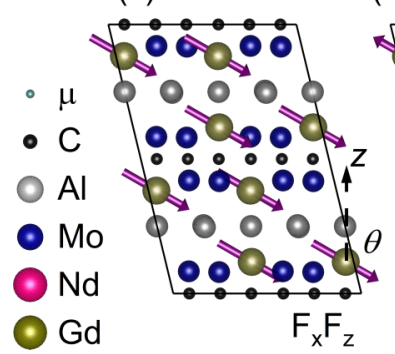

(b)

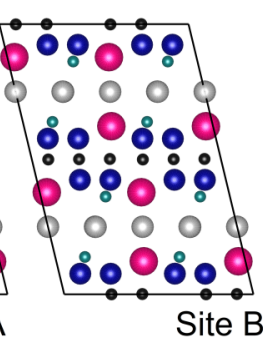

(d)

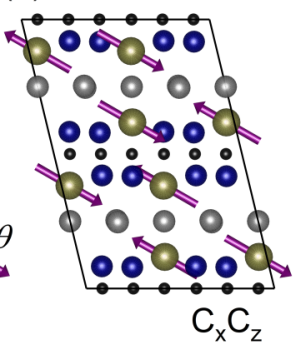

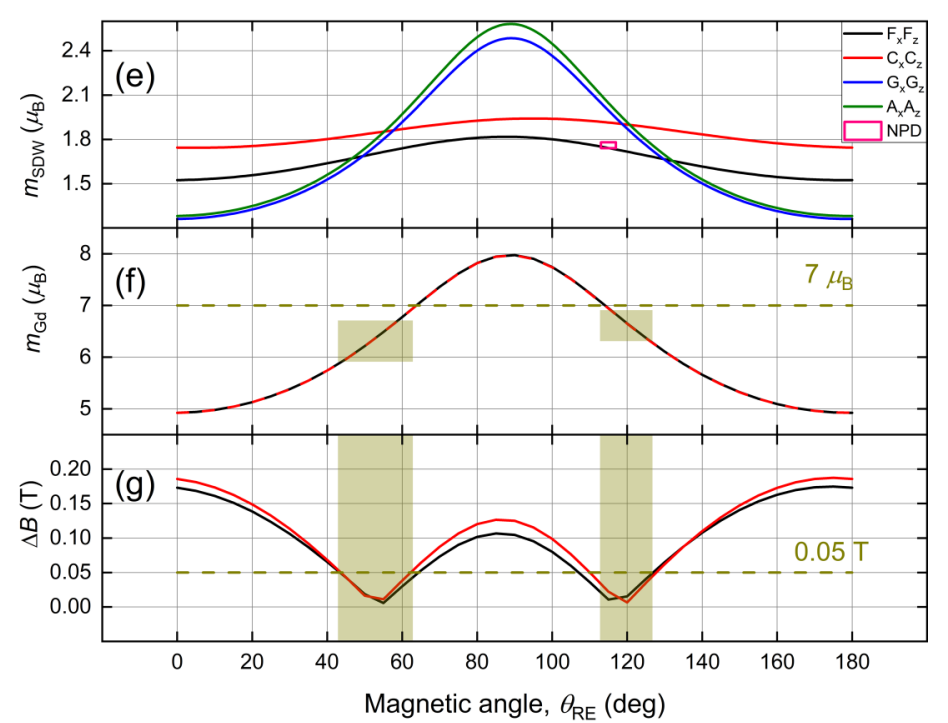

FIG. 4. Lowest energy muon $(\mu)$ site $A(a)$ and muon site $B(b)$ shown as dark cyan spheres. (c) The possible magnetic $F_{x} F_{z}$ configuration for $\mathrm{RE}=\mathrm{Gd}$ in the $a-c$ plane and the geometric definition of the magnetic angle $\theta$. (d) The possible magnetic $\mathrm{C}_{\mathrm{x}} \mathrm{C}_{\mathrm{z}}$ configuration for $\mathrm{RE}=\mathrm{Gd}$. Both configurations in (c) and (d) have a propagation vector of $\mathbf{k}_{\mathrm{Gd}}=(0,0.5,0)$. (e) Calculated SDW amplitude for $\mathrm{RE}=$ $\mathrm{Nd}$ as a function of moments' direction $\theta_{\mathrm{RE}}$ for different spin configurations assuming muon site A. The NPD result (Table II) is given as a pink rectangle, indicating uncertainties on the refined value. (f) Calculated physical magnetic moment for $\mathrm{RE}=\mathrm{Gd}$ as a function of magnetic moment direction $\theta_{\mathrm{RE}}$ for $\mathbf{k}_{\mathrm{Gd}}=(0,0.5,0)$ and assuming all magnetic moments in the unit cell have the same magnitude. (g) Calculated magnetic field inhomogeneity among muon sites in the unit cell for RE $=$ Gd. Results consistent with observed $\mu$ SR data in (f) and (g) are highlighted using dark yellow rectangles. 
In $\mathrm{RE}=\mathrm{Nd}$, two candidate muon sites are obtained. They are labeled as A [Fig. 4(a)] with fractional coordinates $(0.438$, $0.111,0.250)$ and $\mathrm{B}$ [Fig. 4(b)] with fractional coordinates $(0.343,0.114,0.362)$. The two sites have nearly degenerate energy ( $\approx 20 \mathrm{meV}$ difference). In contrast, for $\mathrm{RE}=\mathrm{Gd}$, site A with coordinates $(0.448,0.087,0.250)$ has the lowest energy by a margin of more than $100 \mathrm{meV}$. DFT calculations for other REs yield site A as the lowest energy site as well.

After the determination of candidate muon sites in RE $=$ $\mathrm{Nd}$, the magnetic moment is calculated by assuming a given magnetic structure. The magnetic field at the muon site is determined by dipolar contributions from a $13 \times 13 \times 13$ unit cell grid centered on the muon site. Following the refinement strategy of the NPD data, four magnetic configurations, based on the basis vectors, namely, $F_{x} F_{z}$ [Fig. 4(c)], $G_{x} G_{z}, C_{x} C_{z}$ [Fig. 4(d)], and $A_{x} A_{z}$, are considered. For each configuration, the moments are constrained to lie in the $a-c$ plane. A magnetic moment at site $i$ is then given by

$$
\mathbf{m}_{i}=m_{\mathrm{SDW}}\left(\sin \theta_{\mathrm{RE}} \hat{\mathbf{x}}+\cos \theta_{\mathrm{RE}} \hat{\mathbf{z}}\right) \cos \left(2 \pi \mathbf{k}_{\mathrm{RE}} \cdot \mathbf{r}_{i}+\psi_{\mathrm{RE}}\right),
$$

where $m_{\mathrm{SDW}}$ is the SDW amplitude, $\theta_{\mathrm{RE}}$ is the magnetic moment angle relative to the Cartesian $z$ axis [Fig. 4(c)], $\mathbf{k}_{\mathrm{RE}}$ is the propagation vector, $\mathbf{r}_{\mathrm{i}}$ is the position of the $i$ th ion, and $\psi_{\mathrm{RE}}$ is a global magnetic phase. Since the magnetic structure of $\mathrm{RE}=\mathrm{Nd}$ is incommensurate, $\psi_{\mathrm{Nd}}$ is arbitrary and can be set to zero. A scan over $\theta_{\mathrm{Nd}}$ is performed and $m_{\mathrm{SDW}}$ corresponding to each $\theta_{\mathrm{Nd}}$ is then calculated by matching the calculated magnetic field at the muon site to the observed field [Fig. 3(c)]. Among the resulting $m_{\mathrm{SDW}}-\theta_{\mathrm{Nd}}$ curves for muon site A [Fig. 4(e)], only the $\mathrm{F}_{\mathrm{x}} \mathrm{F}_{\mathrm{Z}}$ configuration contains a $\left(m_{\mathrm{SDW}}, \theta_{\mathrm{Nd}}\right)$ pair, which matches the results obtained from NPD [Fig. 4(e), pink rectangle]. For muon site B, all calculated curves (Fig. S2) [25] require $m_{\mathrm{SDW}}>2 \mu_{\mathrm{B}} / \mathrm{Nd}$ and thus do not intersect the NPD result. This indicates that even in the $\mathrm{RE}=\mathrm{Nd}$ compound, site A seems to be the stable muon site and, it is concluded that site $\mathrm{A}$ is the most likely muon site candidate in all $\left(\mathrm{Mo}_{2 / 3} \mathrm{RE}_{1 / 3}\right)_{2} \mathrm{AlC}$.

Having confirmed the muon site and magnetic field calculations on $\mathrm{RE}=\mathrm{Nd}$, a similar process for determining possible magnetic structures in $\mathrm{RE}=\mathrm{Gd}$ is performed. To limit the number of candidate magnetic structures, several constraints are placed on the magnetic moments in the $\mathrm{RE}=\mathrm{Gd}$ unit cell. First, since there is only a single dominant frequency in the $\mathrm{RE}=\mathrm{Gd}$ field spectrum, it can be safely assumed that the periodicity of its magnetic structure is commensurate with the crystal lattice. Because all $\left(\mathrm{Mo}_{2 / 3} \mathrm{RE}_{1 / 3}\right)_{2} \mathrm{AlC}$ phases in this study are isostructural, it is reasonable to assume that the propagation vector for $\mathrm{RE}=\mathrm{Gd}$ has the same form as for the other compounds, i.e., $\left(0, k_{\mathrm{Gd}}, 0\right)$. Moreover, a single magnetic field requires $k_{\mathrm{Gd}}$ to be either 0 or $1 / 2$, as the magnetic moment magnitude must stay constant between adjacent unit cells.
Second, the possible spin configurations in the unit cell are limited to the four configurations tested for $\mathrm{RE}=\mathrm{Nd}$. These configurations ensure that atoms 1-4 in the unit cell [Fig. 2(e)] have the same magnetic moment, as required to produce a single magnetic field in all muon sites, and are compatible with both $k_{\mathrm{Gd}}=0$ and $k_{\mathrm{Gd}}=1 / 2$ periodicities. Third, since the magnetic moments in compounds with $\mathrm{RE}=\mathrm{Nd}, \mathrm{Tb}, \mathrm{Ho}$, and $\mathrm{Er}$ are oriented within the $a-c$ plane, the same constraint is imposed on $\mathrm{RE}=\mathrm{Gd}$. The available fitting parameters are therefore $m_{\mathrm{SDW}}, \theta_{\mathrm{Gd}}$, and $\psi_{\mathrm{Gd}}$.

Attempts to directly fit $m_{\mathrm{SDW}}, \theta_{\mathrm{Gd}}$, and $\psi_{\mathrm{Gd}}$ by calculating the resulting asymmetry and minimizing the squared differences between calculated and observed values yield unstable results. Therefore a brute force scan approach is employed. For $\mathbf{k}_{\mathrm{Gd}}=(0,0,0), \psi_{\mathrm{Gd}}$ has no physical meaning and is set to zero. Scans of $\theta_{\mathrm{Gd}}$ are performed for representations $\Gamma_{1}$, $\Gamma_{3}$, and $\Gamma_{4}$ (Table IV), since the FM representation $\Gamma_{2}$ can be ruled out from magnetization measurements [5]. Values for $\theta_{\mathrm{Gd}}$, which are consistent with the magnetic field distribution of $\mathrm{RE}=\mathrm{Gd}$ [Fig. 3(d)], are selected by requiring (i) $m_{\mathrm{Gd}} \leqslant 7 \mu_{\mathrm{B}}$, i.e., lower than the $\mathrm{Gd}^{3+}$ free ion moment [32], and (ii) $\Delta B \leqslant 0.05 \mathrm{~T}$, where the field inhomogeneity $\Delta B$ is the difference between the maximal and minimal field values among the muon sites, and $0.05 \mathrm{~T}$ is the FWHM of the observed magnetic field distribution obtained by fitting it with a Lorentzian peak shape [Fig. 3(d), solid line]. Results consistent with conditions (i) and (ii) are found for $\Gamma_{1}$ and $\Gamma_{3}$ with Gd magnetic moment values ranging between $5.2 \mu_{\mathrm{B}}$ and $7 \mu_{\mathrm{B}}$. For $\mathbf{k}_{\mathrm{Gd}}=(0,0.5,0)$ it is assumed that all magnetic moments in the $\left(\mathrm{Mo}_{2 / 3} \mathrm{RE}_{1 / 3}\right)_{2} \mathrm{AlC}$ unit cell have equal moments (i.e., $\psi_{\mathrm{Gd}}= \pm 45^{\circ}$ ) for simplicity. The physical moment on the $\mathrm{Gd}$ atom is denoted as $m_{\mathrm{Gd}}=m_{\mathrm{SDW}} \cos \psi_{\mathrm{Gd}}$. Calculations for a general $\psi_{\mathrm{Gd}}$ are treated in Sec. III D. For each candidate structure a scan over $\theta_{\mathrm{Gd}}$ is performed in the range $0^{\circ} \leqslant \theta_{\mathrm{Gd}} \leqslant 180^{\circ}$ [Fig. 4(f)]. For each $\theta_{\mathrm{Gd}}$ the average magnetic field over eight muon sites in the unit cell $\bar{B}$ and $\Delta B$ [Fig. $4(\mathrm{~g})]$ is computed. $m_{\mathrm{Gd}}$ is calculated by requiring $\bar{B}=1.426 \mathrm{~T}$, which is the observed average magnetic field (Table III). It is found that only configurations belonging to $\Gamma_{2}$ (Table IV) can satisfy conditions (i) and (ii) simultaneously, and the resulting magnetic moment ranges between 6 and $7 \mu_{\mathrm{B}}$.

\section{Muon spin rotation on $\left(\mathrm{Mo}_{2 / 3} \mathrm{Gd}_{1 / 3}\right)_{2} \mathrm{AIC}$ single crystals}

Time dependence of the muon asymmetry is recorded using two perpendicular pairs of detectors (Fig. 5, inset). The first, forward-backward (FB) detectors, measure the muon polarization along the muon momentum direction ( $z$ axis). The second, up-down (UD) detectors, measure the muon asymmetry perpendicular to the muon momentum ( $x$ axis). The muon-site-averaged asymmetry $\bar{A}$ along each pair of detectors is calculated in Appendix A with the results

$$
\begin{gathered}
\bar{A}_{\mathrm{UD}}(t)=\frac{1}{4} A_{\mathrm{UD}} \sin \zeta \frac{1}{8} \sum_{i=1}^{8}\left[e^{-\lambda_{\mathrm{F}} t} \cos \left(\gamma_{\mu} B_{i} t\right)\left(3+\cos 2 \eta_{i}\right)+2 e^{-\lambda_{S} t} \sin ^{2} \eta_{i}\right]+A_{\mathrm{UD}}^{\mathrm{BG}}(\zeta) \\
\bar{A}_{\mathrm{FB}}(t)=A_{\mathrm{FB}} \cos \zeta \frac{1}{8} \sum_{i=1}^{8}\left[e^{-\lambda_{F} t} \cos \left(\gamma_{\mu} B_{i} t\right) \sin ^{2} \eta_{i}+e^{-\lambda_{S} t} \cos ^{2} \eta_{i}\right]+A_{\mathrm{FB}}^{\mathrm{BG}}(\zeta)
\end{gathered}
$$


TABLE IV. Scan results for possible magnetic configurations of $\left(\mathrm{Mo}_{2 / 3} \mathrm{Gd}_{1 / 3}\right)_{2} \mathrm{AlC}$ for two possible propagation vectors. The results are given as ranges for $\mathbf{k}_{\mathrm{Gd}}=(0,0,0)$ and as bounds for $\mathbf{k}_{\mathrm{Gd}}=(0,0.5,0)$. Parameters listed for modes of $\mathbf{k}_{\mathrm{Gd}}=(0,0.5,0)$ are for the magnetic structures of highest symmetry (see text). Dash indicates no suitable pairs $\left(\theta_{\mathrm{Gd}}, \psi_{\mathrm{Gd}}\right)$ found.

\begin{tabular}{|c|c|c|c|c|c|}
\hline Propagation vector, $\mathbf{k}_{\mathrm{Gd}}$ & Representation & Mode & $m_{\mathrm{Gd}}\left(\mu_{\mathrm{B}}\right)$ & $\theta_{\mathrm{Gd}}(\mathrm{deg})$ & $\psi_{\mathrm{Gd}}(\mathrm{deg})$ \\
\hline \multirow[t]{3}{*}{$(0,0,0)$} & $\Gamma_{1}$ & $\mathrm{G}_{\mathrm{x}} \mathrm{G}_{\mathrm{z}}$ & $5.2-7$ & $\begin{array}{c}0-48 \\
130-180\end{array}$ & 0 \\
\hline & $\Gamma_{3}$ & $\mathrm{~A}_{\mathrm{x}} \mathrm{A}_{\mathrm{z}}$ & $5.2-7$ & $\begin{array}{c}0-48 \\
130-180\end{array}$ & 0 \\
\hline & $\Gamma_{4}$ & $\mathrm{C}_{\mathrm{x}} \mathrm{C}_{\mathrm{z}}$ & - & - & - \\
\hline & & $\mathrm{A}_{\mathrm{x}} \mathrm{A}_{\mathrm{z}}$ & - & - & - \\
\hline & $\Gamma_{2}$ & $\mathrm{~F}_{\mathrm{X}} \mathrm{F}_{\mathrm{z}}$ & $6.3 \pm 0.4$ & $53 \pm 10$ & $-45^{\mathrm{b}}$ \\
\hline & & & $6.6 \pm 0.3$ & $120 \pm 7$ & \\
\hline & & $\mathrm{C}_{\mathrm{x}} \mathrm{C}_{\mathrm{z}}$ & $6.3 \pm 0.4$ & $53 \pm 9$ & $45^{\mathrm{b}}$ \\
\hline & & & $6.6 \pm 0.3$ & $121 \pm 7$ & \\
\hline
\end{tabular}

a Not considered.

${ }^{\mathrm{b}}$ Constrained to obtain equal moments on Gd atoms.

where $A_{\mathrm{UD}}$ and $A_{\mathrm{FB}}$ are the respective total detector asymmetries, $\zeta$ and $\eta$ are the angles of the initial muon spin and internal magnetic field relative the $z$ axis, respectively, and $A^{\mathrm{BG}}$ represents a background signal from muons stopped in the sample holder or in the glue between the crystallites. The index $i$ denotes the eight muon sites in the $\left(\mathrm{Mo}_{2 / 3} \mathrm{RE}_{1 / 3}\right)_{2} \mathrm{AlC}$ unit cell [Fig. 4(a)]. The magnetic field $B$ and angle $\eta$ are calculated from the magnetic structure parameters $m_{\mathrm{SDW}}, \theta_{\mathrm{Gd}}$, and $\psi_{\mathrm{Gd}}$ as a sum of the dipolar fields.

BT asymmetry of the $\left(\mathrm{Mo}_{2 / 3} \mathrm{Gd}_{1 / 3}\right)_{2} \mathrm{AlC}$ single-crystal sample is shown in Fig. 5, after background subtraction, for a number of muon spin angles $\zeta$. To find magnetic structures, which are consistent with the $\zeta$ dependence of the asymmetry, the powder data is first analyzed for the general $\psi_{\mathrm{Gd}}$ case. From this point on we focus only on the case of $\mathbf{k}_{\mathrm{Gd}}=(0,0.5,0)$, as it is closer to the $\mathbf{k}$ vectors of other REs and is therefore more likely to be the correct option. Pairs of $\left(\theta_{\mathrm{Gd}}, \psi_{\mathrm{Gd}}\right)$ are scanned in the ranges $0^{\circ} \leqslant \theta_{\mathrm{Gd}} \leqslant 180^{\circ}$ and $-90^{\circ} \leqslant \psi_{\mathrm{Gd}} \leqslant 90^{\circ}$ (Fig. 6). A slight change to condition (i) (see Sec. III C) is required for the general case. The physical moments on the Gd atoms are given by $m_{\mathrm{SDW}} \cos \psi_{\mathrm{Gd}}$ and $m_{\mathrm{SDW}} \sin \psi_{\mathrm{Gd}}$, and condition (i) is changed to $\max \left(\left|m_{\mathrm{SDW}} \cos \psi_{\mathrm{Gd}}\right|,\left|m_{\mathrm{SDW}} \sin \psi_{\mathrm{Gd}}\right|\right) \leqslant 7 \mu_{\mathrm{B}}$. Only modes belonging to $\Gamma_{2}$ are consistent with (i) and (ii). For each mode conditions (i) and (ii) are obeyed in four branches in the $\theta_{\mathrm{Gd}}-\psi_{\mathrm{Gd}}$ plane (Fig. 6). In general, the magnetic moment is found to vary between 5 and $7 \mu_{\mathrm{B}}, \theta_{\mathrm{Gd}}$ varies between $0^{\circ}-60^{\circ}$ or $110^{\circ}-180^{\circ}$, while $\psi_{\mathrm{Gd}}$ is centered around $-45^{\circ}$ and $50^{\circ}$ for $F_{x} F_{z}$ and around $-40^{\circ}$ or $45^{\circ}$ for $C_{x} C_{z}$.

To perform a fit to the single-crystal data, initial magnetic structure parameters are selected from one of the four branches in Fig. 6 as a starting guess for the fit. Equations (5) and (6) are fitted to the data sets of both detector pairs and all measured muon spin angles simultaneously. The fit parameters are constrained with conditions (i) and (ii) to stay consistent with the powder results. Different initial magnetic configurations from each branch are tested to check the stability of the fit. The final fit results are shown as $95 \%$ confidence ellipses in Fig. 6 and solid lines in Fig. 5. Stable results are found in three out of the four branches for each magnetic configuration. The resulting magnetic moment for each fit result lies in the range 6.94-7 $\mu_{\mathrm{B}}$, which matches the free ion moment of $\mathrm{Gd}^{3+}$. The fitted values of $\psi_{\mathrm{Gd}}$ in the branches that include $\psi_{\mathrm{Gd}}= \pm 45^{\circ}$ are found in the range $\left|\psi_{\mathrm{Gd}}\right|=45^{\circ} \pm 1^{\circ}$ for both magnetic configurations. This result agrees with our estimate for the most likely magnetic structure based on powder data alone, with the assumption that the magnetic moments in the unit cell have equal magnitudes (Table IV). Thus these results put further constraints on the possible magnetic structures of $\left(\mathrm{Mo}_{2 / 3} \mathrm{Gd}_{1 / 3}\right)_{2} \mathrm{AlC}$.

\section{E. Temperature evolution \\ 1. Neutron diffraction}

To obtain the temperature evolution of the magnetic and crystal structures of $\left(\mathrm{Mo}_{2 / 3} \mathrm{RE}_{1 / 3}\right)_{2} \mathrm{AlC}$ from the NPD data, a number of sequential refinements are performed. The initial refinement contains no constraints on the diffraction pattern parameters. The instrumental parameters (zero-shift and sample offset), scale factors, and lattice constants of all phases, atomic positions of the main phase, and magnetic structure parameters are refined. After the initial refinement, the temperature evolution of each parameter is examined, and any parameter that shows no significant and systematic change with temperature is fixed to be the weighted average of all refined temperatures. The final refinement consists of the LPs, magnetic moment (magnitude and direction), and propagation vector refined, while all other parameters are fixed using their corresponding weighted averages. In the high-resolution NPD data of $\mathrm{RE}=\mathrm{Er}$ and Ho, some atomic positions display a nontrivial temperature dependence and are refined as well.

The temperature evolution of $\mathbf{k}_{\mathrm{RE}}$ (Fig. 7) is observed for $\mathrm{RE}=\mathrm{Nd}$ and $\mathrm{Tb}$, which is characteristic of incommensurate SDWs, while for $\mathrm{RE}=\mathrm{Ho}$ and $\mathrm{Er}, \mathbf{k}_{\mathrm{RE}}$ stays constant with a value close to $2 / 3$, indicating a commensurate SDW. The $b$ LP (Fig. 7) shows the strongest magnetostriction, which is closely correlated with $T_{\mathrm{N}}$ with up to an $0.08 \%$ expansion for $\mathrm{RE}=$ $\mathrm{Tb}$. 


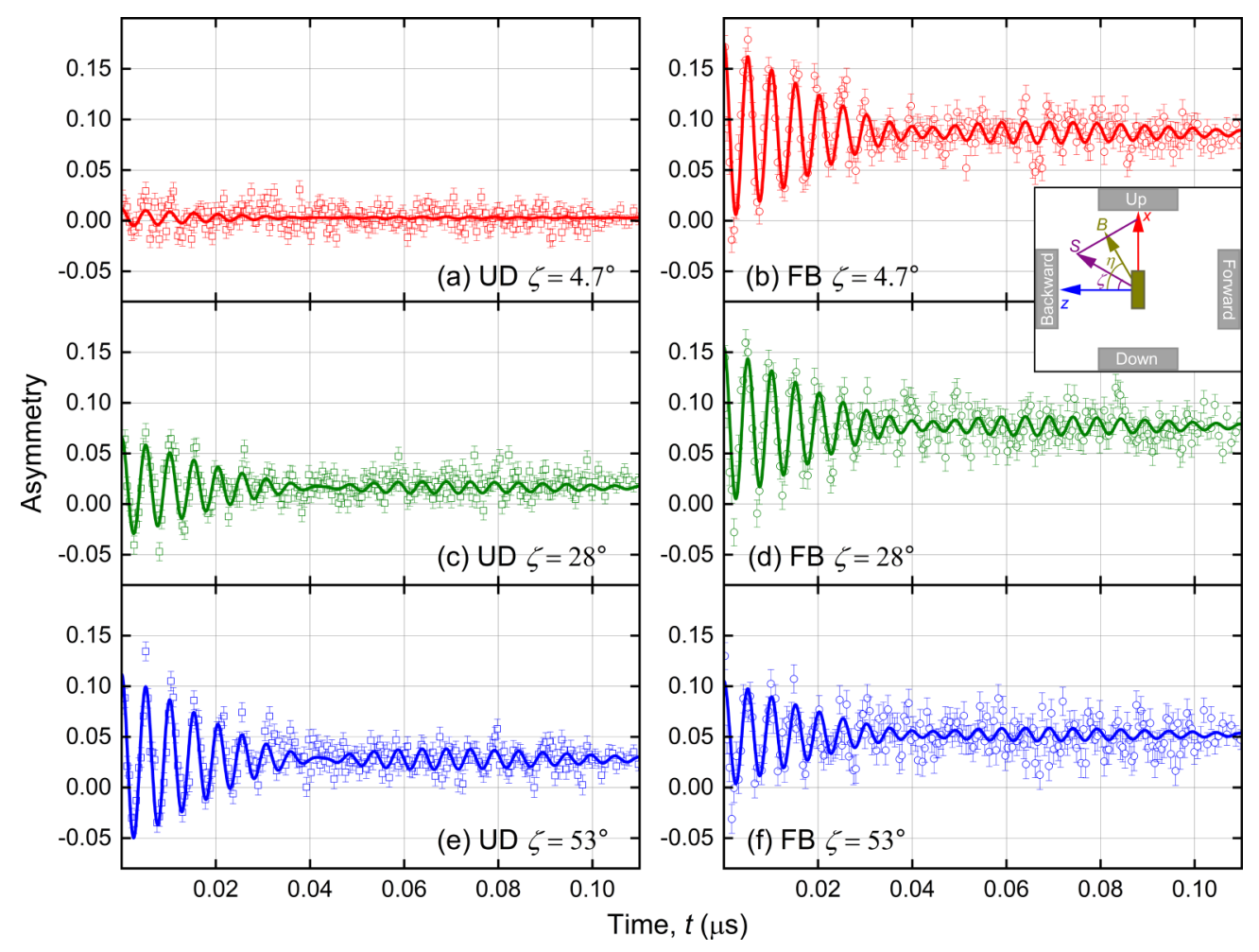

FIG. 5. Time dependence of the BT asymmetry (after background subtraction) for different initial muon spin angles $\zeta$. Left column shows the up-down detectors and right column, the forward-backward detectors. Solid lines are fits of Eqs. (5) and (6) to the data. Error bars denote statistical $(1 \sigma)$ errors. Inset shows a schematic representation of the detector geometry in the GPS muon spectrometer. The different pairs of detectors are shown as gray rectangles, the sample as a dark yellow rectangle. Arrows indicate the $x$ and $z$ axes, with the $y$ axis pointing into the page. The magnetic field of the sample is shown as a dark yellow arrow and the direction of the muon spin as a purple arrow. The purple line shows the precession motion of the spin about the magnetic field.

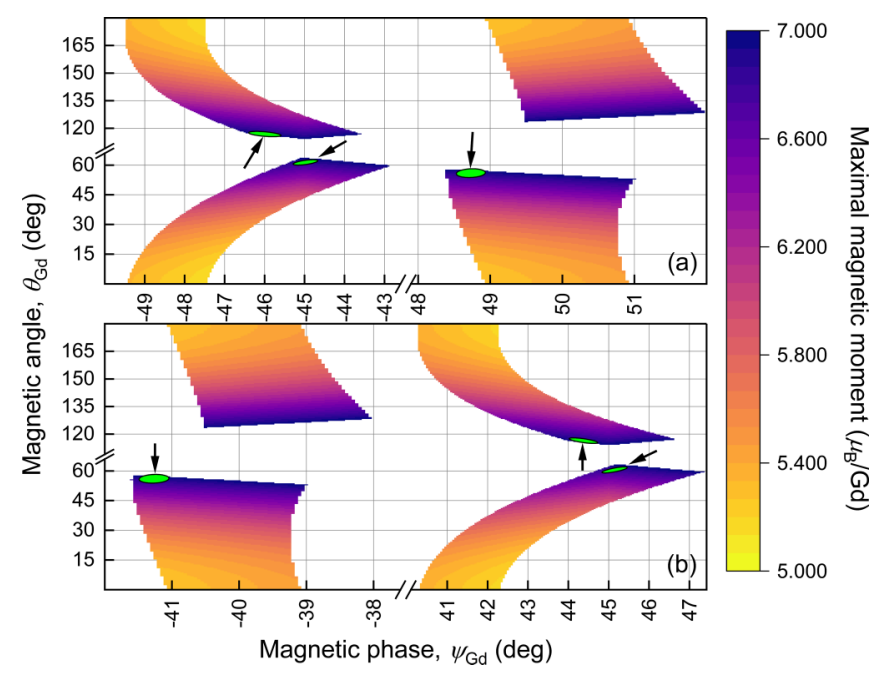

FIG. 6. Calculated maximal magnetic moment for $\left(\mathrm{Mo}_{2 / 3} \mathrm{Gd}_{1 / 3}\right)_{2} \mathrm{AlC}$ as a function of $\psi_{\mathrm{Gd}}$ and $\theta_{\mathrm{Gd}}$. The calculations are performed assuming magnetic configuration (a) $\mathrm{F}_{\mathrm{x}} \mathrm{F}_{\mathrm{z}}$ and (b) $\mathrm{C}_{\mathrm{x}} \mathrm{C}_{\mathrm{z}}$. Colored areas show regions consistent with conditions (i) and (ii) that are required to reproduce the observed magnetic field distribution (see text). Green ellipses, emphasized by arrows, show 95\% confidence regions for magnetic structure parameters obtained by fitting to the $\mu \mathrm{SR}$ data measured from the $\mathrm{RE}=\mathrm{Gd}$ single-crystal sample (see text).
$\mathrm{RE}=\mathrm{Tb}$ displays a magnetic structure which consists of two propagation vectors $\mathbf{k}_{\mathrm{Tb}, 1}=(0,0.5,0)$ and $\mathbf{k}_{\mathrm{Tb}, 2}=$ $(0,0.64,0)$ (Table II). As $T$ increases, reflections belonging

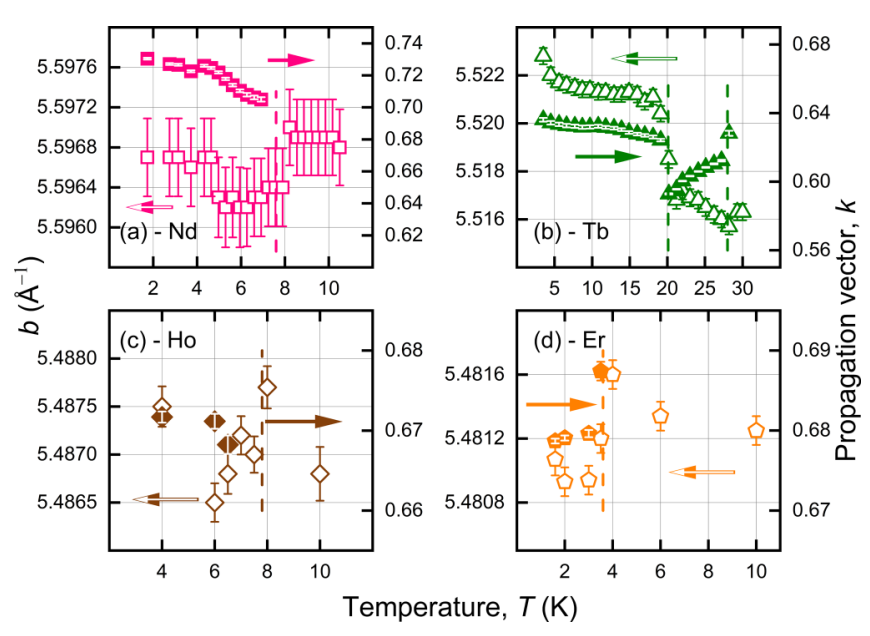

FIG. 7. Temperature evolution of the $b$ lattice parameter (open symbols, left ordinate) and propagation vector $k$ (full symbols, right ordinate) as determined by neutron diffraction. Dashed vertical lines indicate $T_{\mathrm{N}}$ for each phase. Error bars in (b), (c), and (d) indicate statistical $(1 \sigma)$ uncertainties, while error bars in (a) contain a systematic uncertainty from the low fit quality for $\mathrm{RE}=\mathrm{Nd}$. 


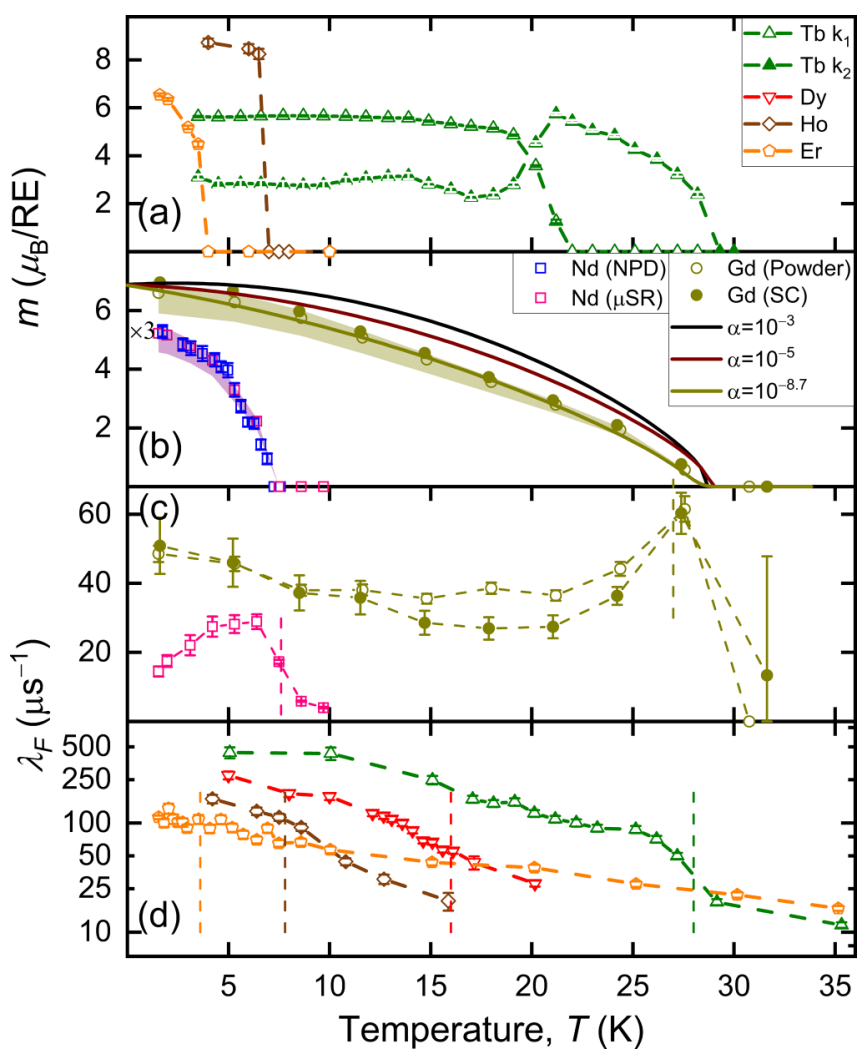

FIG. 8. Temperature evolution of the magnetic moment for $\left(\mathrm{Mo}_{2 / 3} \mathrm{RE}_{1 / 3}\right)_{2} \mathrm{AlC}$ with (a) $\mathrm{RE}=\mathrm{Tb}, \mathrm{Ho}$, and $\mathrm{Er}$ and (b) $\mathrm{RE}=$ $\mathrm{Nd}$ and $\mathrm{RE}=\mathrm{Gd}$. Pink squares in (b) are obtained from $\mu \mathrm{SR}$ by taking $\theta_{\mathrm{Nd}}=115^{\circ}$ as measured by NPD (Table II). Open circles in (b) are calculated for $\theta_{\mathrm{Gd}}=120^{\circ}$. The data for $\mathrm{RE}=\mathrm{Nd}$ in (b) is multiplied by 3 for clarity. Solid lines show the calculated magnetic moment for $\mathrm{RE}=\mathrm{Gd}$ for $\alpha=10^{-3}$ (black line), $\alpha=10^{-5}$ (dark red), and $\alpha=10^{-8.7}$ (dark yellow, see text). Panels (c) and (d) show the temperature evolution of the muon relaxation rate for (c) RE $=\mathrm{Nd}$ and $\mathrm{RE}=\mathrm{Gd}$ and (d) $\mathrm{RE}=\mathrm{Tb}, \mathrm{Dy}, \mathrm{Ho}$, and Er. Dashed vertical lines in (c) and (d) show $T_{\mathrm{N}}$ for each compound. Closed circles in (b) and (c) are obtained from fitting to the single-crystal data. Error bars on symbols represent $1 \sigma$ statistical uncertainties, while systematic uncertainties in the magnetic moments obtained from $\mu$ SR measurements are shown as confidence bands.

to $\mathbf{k}_{\mathrm{Tb}, 1}$ decrease in intensity and show no relative intensity change between reflections. The direction of the $\mathbf{k}_{\mathrm{Tb}, 1}$ magnetic moments is therefore kept fixed. Reflections belonging to $\mathbf{k}_{\mathrm{Tb}, 2}$ do not show any significant changes up to $19 \mathrm{~K}$; however, both relative intensities and positions of the $\mathbf{k}_{\mathrm{Tb}, 2}$ magnetic reflections change above that temperature. Above $20 \mathrm{~K}$, reflections belonging to $\mathbf{k}_{\mathrm{Tb}, 1}$ disappear. The moment configuration of $\mathbf{k}_{\mathrm{Tb}, 2}$ at these temperatures is identified by calculating the difference pattern and testing possible combinations of basis vectors. It is found that the magnetic structure belonging to $\mathbf{k}_{T b, 2}$ changes from $C_{x} F_{z}$ to $C_{x} C_{z}$ (Fig. S3) [25] above $20 \mathrm{~K}$ and therefore refinement consists of the $\mathrm{C}_{\mathrm{x}} \mathrm{C}_{\mathrm{z}}$ structure at these temperatures.

Temperature evolution of the refined magnetic moments of $\mathrm{RE}=\mathrm{Tb}, \mathrm{Ho}$, and Er are shown in Fig. 8(a). For $\mathrm{RE}=\mathrm{Tb}$, the magnetic moments of both propagation vectors reach saturation (upon cooling) below $15 \mathrm{~K}$ and switch magnitudes above
$19 \mathrm{~K}$. Slightly below $T_{\mathrm{N}}$, the magnetic reflections in $\mathrm{RE}=$ $\mathrm{Ho}$ and Er become indistinguishable from the background due to strong diffuse magnetic scattering which results from short-range magnetic order in the sample. The abrupt drop in the magnetic moments of RE $=\mathrm{Ho}$ and $\mathrm{Er}$ in [upon heating, Fig. 8(a)] signifies the limit of detection of long-range magnetic order in the respective diffractometers.

The observed magnetic reflections of $\mathrm{RE}=\mathrm{Nd}$ are too weak to be properly refined using the full crystallographic and magnetic patterns. The magnetic structure of this compound is therefore refined using the difference pattern for all temperatures and then kept fixed in the refinement of the full pattern. Due to the low fit quality of the $\mathrm{RE}=\mathrm{Nd}$ magnetic structure, a more accurate temperature evolution of its magnetic moment [Fig. 8(b)] is obtained by taking the integrated intensity of the $(-11-k 4)$ reflection (Fig. S1) [25]. This reflection is chosen, as it is strong and does not overlap with any nearby reflections. The magnetic moment of $\mathrm{RE}=\mathrm{Nd}$ is then determined by normalizing the integrated intensity to the magnetic moment obtained from refinement at $1.5 \mathrm{~K}$.

\section{Muon spin rotation}

Fitting of the muon asymmetry parameters to the temperature-dependent muon asymmetry data of powder samples is first performed independently for all measured temperatures, including a fit of the detector efficiency ratio parameter. After examining the temperature behavior of the fitted parameters, a global fit is performed using Eqs. (1)-(3) with a global detector efficiency parameter and a constraint $A_{F}+A_{S}=A_{0}$, with $A_{0}$ being a global fit parameter as well. For temperatures above which no oscillations are observed, $B$ and $\phi$ are constrained to be 0 . Eq. (7) is fitted to the $\left(\mathrm{Mo}_{2 / 3} \mathrm{Gd}_{1 / 3}\right)_{2} \mathrm{AlC}$ single-crystal temperature-dependent data with the constraint $A_{F}{ }^{D}+A_{S}{ }^{D}+A_{B G}{ }^{D}=A_{0}{ }^{D}$, where $A_{0}{ }^{D}$ is a global fit parameter:

$$
\begin{aligned}
A^{D}(T, t)= & A_{F}^{D}(T) \cos \left[\gamma_{\mu} B(T) t+\phi^{D}\right] e^{-\lambda_{F}(T) t} \\
& +A_{S}^{D}(T) e^{-\lambda_{S}(T) t}+A_{\mathrm{BG}}^{D} e^{-\lambda_{\mathrm{BG}}(T) t} .
\end{aligned}
$$

Here $D$ denotes the pair of detectors UD or FB. The fit is first performed on all measured data sets individually. Then a second fit is performed with parameters not depending on $T$ being shared between data sets, while parameters not depending on $D$ being shared between data sets of different detector pairs. Figure 8(b) presents the temperature evolution of the magnetic moment in the two magnets that show long time stability, namely, $\mathrm{RE}=\mathrm{Nd}$ and $\mathrm{Gd}$. For clarity, the moment size of $\mathrm{RE}=\mathrm{Nd}$ is multiplied by 3 . The shaded regions represent confidence bands on the $\mu$ SR moment size calculation. In $\mathrm{RE}=\mathrm{Nd}$ good agreement is observed between $\mathrm{NPD}$ and $\mu \mathrm{SR}$ data. Clearly, $\mathrm{RE}=\mathrm{Gd}$ is a stronger magnet in terms of moment size. When the temperature dependence of the single-crystal $\mathrm{Gd}$ magnetic moment and relaxation rate [Figs. 8(b) and 8(c), full circles] is compared with the results from the powder sample [Figs. 8(b) and 8(c), open circles] good agreement is observed. This indicates that the magnetic structure of $\mathrm{RE}=\mathrm{Gd}$ does not depend on the preparation method or sample type.

The temperature dependence of the magnetization of $\mathrm{RE}=$ Gd is analyzed by fitting an average moment $\left\langle S_{z}\right\rangle$ calculation, 
based on the Hamiltonian of a layered AFM,

$$
H=J\left(\sum_{i, \delta_{\|}} \mathbf{S}_{i} \cdot \mathbf{S}_{i+\delta_{\|}}+\alpha \sum_{i, \delta_{\perp}} \mathbf{S}_{i} \cdot \mathbf{S}_{i+\delta_{\perp}}\right),
$$

using Schwinger Boson mean-field theory (SBMFT) [33-36] to the data. More details can be found in Appendix B. In Eq. (8), $\delta_{\|}$denotes in-plane nearest neighbors and $\delta_{\perp}$ denotes out-of-plane nearest neighbors. The fit parameters and their best values are $\alpha=10^{-8.7 \pm 0.7}, m_{0}=6.9 \pm 0.3 \mu_{\mathrm{B}} / \mathrm{Gd}$, and $T_{\mathrm{N}}=29 \pm 1 \mathrm{~K}$, where $m_{0}$ is the calculated moment at $T=$ 0 and $T_{\mathrm{N}}$ is the calculated Néel temperature. The magnetic exchange interaction strength is $J=2.38 \pm 0.03 \mathrm{meV}$. The best fit is presented in Fig. 8(b) by the dark yellow solid line. The fit function is weakly sensitive to $\alpha$ and only its order of magnitude can be determined. Two additional calculations for $\alpha=10^{-3}$ and $10^{-5}$ are added to demonstrate that as anisotropy decreases, $m(T)$ becomes $T$ independent at low temperature, in contrast to the data. The smallness of $\alpha$ supports the $2 \mathrm{D}$ nature of the $\mathrm{RE}=\mathrm{Gd}$ magnet. In contrast, the magnetic moment of $\mathrm{RE}=\mathrm{Tb}$ shows no $T$ dependence below $15 \mathrm{~K}$, which is consistent with a $3 \mathrm{D}$ behavior. $\mathrm{RE}=$ Er resembles anisotropic behavior as in Fig. 8(b), while the relatively high $\mathrm{BT}$ for $\mathrm{RE}=$ Ho does not allow clear determination of the $T \rightarrow 0$ behavior.

The muon relaxation rates in $\mathrm{RE}=\mathrm{Nd}$ and Gd in Fig. 8(c) show classical spin lattice relaxation peak at, or close to, $T_{\mathrm{N}}$, where the spin fluctuations are slow enough and their amplitude is large enough to effectively relax the muon polarization. For heavier REs, however, the situation is drastically different [Fig. 8(d)]. The relaxation rate in $\mathrm{RE}=\mathrm{Tb}$ is nearly five times larger than in $\mathrm{RE}=\mathrm{Gd}$ and shows a monotonic increase with decreasing temperature. Similar trends can be observed in the remaining compounds as well. The fast relaxing component in the muon asymmetry indicates that the magnetic fluctuations slow down to within the $\mu$ SR time frame window of 10 ps-1 $\mu$ s [37,38]. The volume fractions of the fast and slow components (Fig. S4 in the Supplemental Material [25]) in $\mathrm{RE}=\mathrm{Gd}$ show a constant value of $2 / 3$ and $1 / 3$, respectively, up to $T_{\mathrm{N}}$, above which the data is described by a single decay rate. This is consistent with a static magnetic field in $\mathrm{RE}=$ Gd for all $T<T_{\mathrm{N}}$. For other REs, the volume fraction of the fast component gradually decreases while the volume fraction of the slow component increases with increasing temperature, signaling the gradual increase in magnetic disorder. Below $T_{\mathrm{N}}$, the volume fractions of both components stay nearly constant, which, when combined with the temperature dependence of the relaxation rate [Figs. 8(c) and 8(d)], gives another indication for the gradual freezing of the magnetic structure.

\section{DISCUSSION}

The possible magnetic structures of RE $=$ Gd [Figs. 4(c) and 4(d)] are found by combining the NPD and $\mu$ SR techniques. This is made possible due to the availability of isostructural compounds with different RE atoms, as well as the similarity in magnetic structures for different REs and the relatively simple magnetic structure found for $\mathrm{RE}=\mathrm{Gd}$. While $\mu$ SR can be used to refine subtle details in magnetic structures $[39,40]$, which cannot be easily determined from

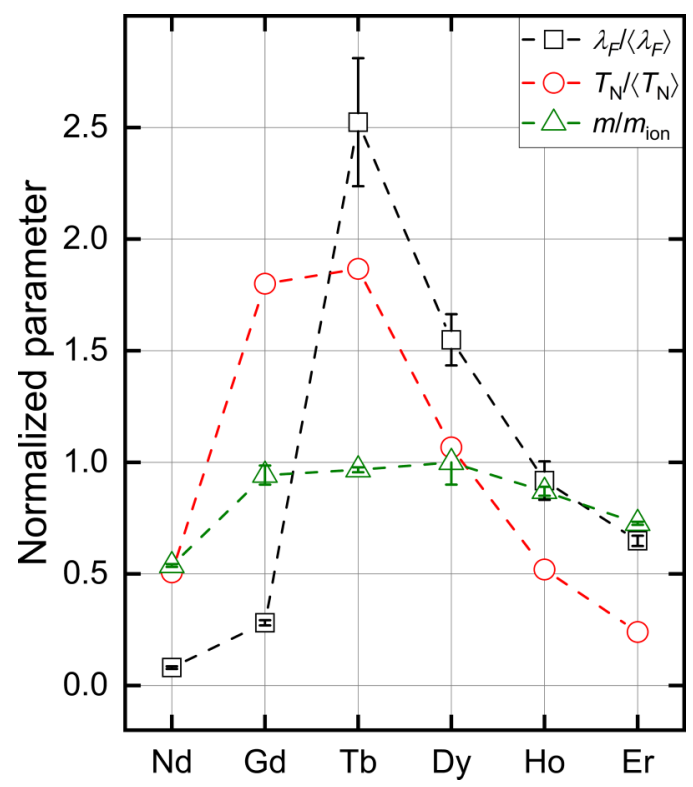

FIG. 9. Dependence of $\mu$ SR relaxation rate $\lambda_{F}$, Néel temperature $T_{\mathrm{N}}$, and magnetic moment $m$ measured at BT (4 K for $m$ of RE $=$ Ho, and $1.5 \mathrm{~K}$ for the rest) on the RE element in $\left(\mathrm{Mo}_{2 / 3} \mathrm{RE}_{1 / 3}\right)_{2} \mathrm{AlC}$. For comparison, $\lambda_{F}$ and $T_{\mathrm{N}}$ are normalized relative to their average over RE, while $m$ is normalized relative the free ion moment $m_{\text {ion }}$. Error bars for $\lambda_{F}$ and $m$ indicate $1 \sigma$ statistical uncertainties. $T_{\mathrm{N}}$ and $m$ data on $\mathrm{RE}=$ Dy is courtesy of Ref. [24].

neutron measurements alone, the technique requires at least a rough magnetic structure to be known a priori. In this work an application of $\mu \mathrm{SR}$, which can predict a magnetic structure of a compound without any preliminary knowledge on the same compound, is demonstrated.

Temperature evolution of the $\mathrm{RE}=\mathrm{Gd}$ magnetic moment [Fig. 8(b)] is well described by an SBMFT calculation up to and including $T_{\mathrm{N}}$. This agreement indicates that critical fluctuations are either weak or occur in a very small temperature range around $T_{\mathrm{N}}$, as SBMFT does not hold for strong fluctuations [34]. The obtained value for $\alpha$ is smaller than values found for some layered $\mathrm{CuO}_{2}$-based materials [34] and is close to a calculated value of $2 \times 10^{-8}$, which was calculated for $\mathrm{Sr}_{2} \mathrm{CuO}_{2} \mathrm{Cl}_{2}$ assuming magnetic dipole interaction between nearest-neighbor layers [34]. This can indicate that interactions between planes in $\mathrm{RE}=\mathrm{Gd}$ are also of dipolar origin.

When some magnetic properties are plotted against the RE atom size (Fig. 9), similar trends can be observed. $T_{\mathrm{N}} /\left\langle T_{\mathrm{N}}\right\rangle$, $\lambda_{F} /\left\langle\lambda_{F}\right\rangle$ and $m / m_{\text {ion }}$, measured at $1.5 \mathrm{~K}$, where \langle\rangle indicates average over RE and $m_{\text {in }}$ stands for the free ion moment, show a maximum for $\mathrm{RE}=\mathrm{Tb}$ and follow an arc. The similarity in the RE dependence of $m / m_{\text {ion }}$ and $T_{\mathrm{N}} /\left\langle T_{\mathrm{N}}\right\rangle$ suggests that the reduction in the observed magnetic moment is due to temperature and that $1.5 \mathrm{~K}$ is not low enough to obtain the free ion moment for RE with $T_{\mathrm{N}}<10 \mathrm{~K}$. Indeed, the measurement of $\mathrm{RE}=\mathrm{Er}$ at $0.3 \mathrm{~K}$ resulted in a magnetic moment of $7.5 \mu_{\mathrm{B}} / \mathrm{Er}$, bringing it closer to the free ion moment of $9 \mu_{\mathrm{B}}$.

While the muon relaxation observed for $\mathrm{RE}=\mathrm{Tb}$, Ho, and Er may seem contradictory with NPD results at first, the observation of short-range ordering in $\mathrm{RE}=\mathrm{Ho}$ and $\mathrm{Er}$ via 
broadening of the magnetic reflections [Fig. 2(c), inset] is evidence for a different nature in the magnetic ordering of the heavier lanthanides. The temperature dependence of the magnetic correlation length (Fig. S5) [25] is similar to $\lambda_{F}$ and shows that the slowing down of the magnetic fluctuations is accompanied with a gradual increase in the size of ordered magnetic domains.

The nearly one-to-one correspondence between $\lambda_{F}$ and $T_{\mathrm{N}}$ for REs heavier than Gd also indicates that the relaxation of the $\mu$ SR signal in these compounds occurs due to a gradual freezing of the dynamic magnetic structure. As $T_{\mathrm{N}}$ is lowered with increasing RE mass beyond $\mathrm{Gd}$, the magnetic structure slows down less at $1.5 \mathrm{~K}$, resulting in shorter relaxation rates. The RE dependence of $T_{\mathrm{N}}$, however, does not follow the well-known [41] de Gennes factor given by $(g-1)^{2} \times$ $J(J+1)$, where $g$ is the Landé $g$-factor and $J$ is the total angular momentum of the RE ion, which has its maximum for $\mathrm{RE}=\mathrm{Gd}$. This suggests that the magnetic interactions in the $\left(\mathrm{Mo}_{2 / 3} \mathrm{RE}_{1 / 3}\right)_{2} \mathrm{AlC}$ phases are not described by RKKY interactions alone (as may be expected from an RE-based magnet) but contain additional contributions, such as dipolar interactions between neighboring planes as mentioned for $\mathrm{RE}=\mathrm{Gd}$, which are also responsible for the emergence of spin fluctuations and short-range order in the heavy REs.

\section{CONCLUSION}

From a crystal growth point of view, $\left(\mathrm{Mo}_{2 / 3} \mathrm{RE}_{1 / 3}\right)_{2} \mathrm{AlC}$ with $\mathrm{RE}=\mathrm{Gd}$ is the most promising compound for realization of a rare-earth-based, high-moment, 2D magnet out of the compounds studied here. However, as Gd is a strong neutron absorber, the most informative experimental technique in magnetism, namely, NPD, is extremely challenging. Therefore, information from NPD on sister compounds was combined with information gained from $\mu \mathrm{SR}$ on all compounds in order to determine for all variants the most likely magnetic structures and their properties. $\left(\mathrm{Mo}_{2 / 3} \mathrm{Gd}_{1 / 3}\right)_{2} \mathrm{AlC}$ is found to be a stable commensurate AFM with the highest $T_{\mathrm{N}}=29 \pm 1 \mathrm{~K}$ of the series, a high magnetic moment of $6.5 \pm 0.5 \mu_{\mathrm{B}} / \mathrm{Gd}$, with one of two possible structures shown in Figs. 4(c) and 4(d), and magnetic anisotropy smaller than $10^{-8}$. This compound shows potential in providing new magnetic 2D derivatives for the field of van der Waals heterostructures.

\section{ACKNOWLEDGMENTS}

The author is grateful to Professor H. Shaked for discussions and help with analysis of the NPD data. A.P., A.K., D.P., O.R., and E.N.C. acknowledge the support of the Israel Atomic Energy Commission Pazy Foundation grant. J.R. acknowledges support from the Knut and Alice Wallenberg (KAW) Foundation and the Flag-ERA JTC 2017 project "MORE-MXenes." Computational support is provided by the NegevHPC project [42]. This work is partly based on experiments performed at the Swiss spallation neutron source SINQ, and the Swiss muon source $(\mathrm{S} \mu \mathrm{S})$, Paul Scherrer Institute, Villigen, Switzerland. Certain commercial equipment, instruments, or materials (or suppliers, or software, ...) are identified in this paper to foster understanding. Such identi- fication does not imply recommendation or endorsement by the National Institute of Standards and Technology, nor does it imply that the materials or equipment identified are necessarily the best available for the purpose.

\section{APPENDIX A}

The geometry of the muon spin experiment is depicted in Fig. 5 (inset). The initial muon spin direction is rotated by an angle $\zeta$ from the $z$ axis in the $x-z$ plane:

$$
\mathbf{S}_{0}=A_{\mathrm{UD}} \sin \zeta \hat{\mathbf{x}}+A_{\mathrm{FB}} \cos \zeta \hat{\mathbf{z}},
$$

while the direction of the magnetic field is denoted using standard spherical coordinates $(\eta, \chi)$ :

$$
\mathbf{B}=B \sin \eta \cos \chi \hat{\mathbf{x}}+B \sin \eta \sin \chi \hat{\mathbf{y}}+B \cos \eta \hat{\mathbf{z}} .
$$

Here, $A_{\mathrm{UD}}$ and $A_{\mathrm{FB}}$ denote the maximal asymmetry along each pair of detectors. The crystal flake is assumed to lie in the $x-y$ plane. Time evolution of the muon spin, given an arbitrary magnetic field, is given by

$$
\begin{aligned}
\mathbf{S}(t ; \zeta, \eta, \chi)= & \mathbf{S}_{\|} e^{-\lambda_{S} t}+\mathbf{S}_{\perp, 1} e^{-\lambda_{F} t} \cos (\gamma|\mathbf{B}| t) \\
& +\mathbf{S}_{\perp, 2} e^{-\lambda_{F} t} \sin (\gamma|\mathbf{B}| t),
\end{aligned}
$$

where $\mathbf{S}_{\|}=\left(\mathbf{S}_{0} \cdot \hat{\mathbf{B}}\right) \hat{\mathbf{B}}$ is the component of the spin, parallel to the magnetic field, $\mathbf{S}_{\perp, 1}=\mathbf{S}_{0}-\mathbf{S}_{\|}$and $\mathbf{S}_{\perp, 2}=\hat{\mathbf{B}} \times \mathbf{S}_{\perp, 1}$ are the two perpendicular components. $\lambda_{F}$ and $\lambda_{S}$ are the relaxation rates of the fast and slow decaying components, respectively. Since the flakes are randomly oriented in the plane, the total evolution of the muon polarization is obtained by averaging over $\chi$ :

$$
\mathbf{S}_{\mathrm{avg}}(t ; \zeta, \eta)=\frac{1}{2 \pi} \int_{0}^{2 \pi} \mathbf{S}(t ; \zeta, \eta, \chi) d \chi
$$

The polarization along each pair of detectors is obtained by projecting $\mathbf{S}_{\text {avg }}$ along the corresponding axes. By plugging Eqs. (A1)-(A3) into Eq. (A4), we obtain

$$
\begin{aligned}
S_{\mathrm{UD}}(t)= & \mathbf{S}_{\mathrm{avg}} \cdot \hat{\mathbf{x}}=\frac{1}{4} A_{\mathrm{UD}} \sin \zeta\left[e^{-\lambda_{F} t} \cos (\gamma B t)(3+\cos 2 \eta)\right. \\
+ & \left.2 e^{-\lambda_{S} t} \sin ^{2} \eta\right], \\
S_{\mathrm{FB}}(t)= & \mathbf{S}_{\mathrm{avg}} \cdot \hat{\mathbf{z}}=A_{\mathrm{FB}} \cos \zeta\left[e^{-\lambda_{F} t} \cos (\gamma B t) \sin ^{2} \eta\right. \\
& \left.+e^{-\lambda_{S} t} \cos ^{2} \eta\right] .
\end{aligned}
$$

Since there are eight muon sites in the unit cell, the final polarization is obtained by averaging over all muon sites, which gives Eqs. (5) and (6).

\section{APPENDIX B}

Calculation of the magnetic moment of $\mathrm{RE}=\mathrm{Gd}$ is performed by using the nearly 2D antiferromagnetic Heisenberg model given in Eq. (8). Details on the derivation of meanfield equations for this Hamiltonian can be found elsewhere [34-36]. The self-consistent mean-field equations which are solved for $h$ and $\Delta$ are

$$
\begin{aligned}
& h=2 \alpha[S+1 / 2-2 K(\Delta, h, t)] \\
& K(\Delta, h, t)+K(\Delta, 0, t)=S+1 / 2,
\end{aligned}
$$


where $h$ is the staggered interlayer mean field, $\alpha$ is the anisotropy, $t=T / J$ is the reduced temperature, $\Delta$ is a variational gap parameter and $S=3.5$ is the $\mathrm{Gd}^{3+}$ spin. $K$ is given by

$$
K(\Delta, h, t)=2.32 \int_{0}^{1} \frac{1+h+\Delta}{\omega(\Delta, h, \gamma)}\left[n(\omega, t)+\frac{1}{2}\right] \rho(\gamma) d \gamma,
$$

with the dispersion $\omega(\Delta, h, \gamma)=2.32\left[(1+h+\Delta)^{2}-\right.$ $\left.\gamma^{2}\right]^{1 / 2}$, Bose occupancy function $n(\omega, t)=\left(e^{\omega / t}-1\right)^{-1}$, and density of states $\rho(\gamma)=\frac{2}{\pi^{2}} \int_{0}^{1}\left[\left(1-t^{2}\right)\left(1-t^{2}+\right.\right.$ $\left.\left.\gamma^{2} t^{2}\right)\right]^{-1 / 2} d t$. The magnetization, which is fit to the $\mu \mathrm{SR}$ data is given by $M=h(t) / 2 \alpha$. The ground-state magnetic moment of Gd, $m_{0}$ is obtained from the calculated $M$ curve at $t=0$, while the transition temperature $T_{N}$ is calculated numerically by finding the lowest $t$, where $M=0$.
[1] B. Huang, G. Clark, E. Navarro-Moratalla, D. R. Klein, R. Cheng, K. L. Seyler, Di. Zhong, E. Schmidgall, M. A. McGuire, D. H. Cobden, W. Yao, D. Xiao, P. Jarillo-Herrero, and X. Xu, Nature (London) 546, 270 (2017).

[2] C. Gong, L. Li, Z. Li, H. Ji, A. Stern, Y. Xia, T. Cao, W. Bao, C. Wang, Y. Wang, Z. Q. Qiu, R. J. Cava, S. G. Louie, J. Xia, and X. Zhang, Nature (London) 546, 265 (2017).

[3] Z. Fei, B. Huang, P. Malinowski, W. Wang, T. Song, J. Sanchez, W. Yao, D. Xiao, X. Zhu, A. F. May, W. Wu, D. H. Cobden, J. H. Chu, and X. Xu, Nat. Mater. 17, 778 (2018).

[4] Y. Deng, Y. Yu, M. Z. Shi, Z. Guo, Z. Xu, J. Wang, X. H. Chen, and Y. Zhang, Science 367, 895 (2020).

[5] Q. Tao, J. Lu, M. Dahlqvist, A. Mockute, S. Calder, A. Petruhins, R. Meshkian, O. Rivin, D. Potashnikov, E. N. Caspi, H. Shaked, A. Hoser, C. Opagiste, R.-M. Galera, R. Salikhov, U. Wiedwald, C. Ritter, A. R. Wildes, B. Johansson, L. Hultman, M. Farle, M. W. Barsoum, and J. Rosen, Chem. Mater. 31, 2476 (2019).

[6] I. Persson, A. el Ghazaly, Q. Tao, J. Halim, S. Kota, V. Darakchieva, J. Palisaitis, M. W. Barsoum, J. Rosen, and P. O. Å. Persson, Small 14, 1703676 (2018).

[7] A. Champagne, F. Ricci, M. Barbier, T. Ouisse, D. Magnin, S. Ryelandt, T. Pardoen, G. Hautier, M. W. Barsoum, and J. Charlier, Phys. Rev. Mater. 4, 013604 (2020).

[8] A. Gkountaras, Y. Kim, J. Coraux, V. Bouchiat, S. Lisi, M. W. Barsoum, and T. Ouisse, Small 16, 1905784 (2020).

[9] J. Rodríguez-Carvajal, J. Phys. B: Condens. Matter 192, 55 (1993).

[10] T. C. Hansen, P. F. Henry, H. E. Fischer, J. Torregrossa, and P. Convert, Meas. Sci. Technol. 19, 034001 (2008).

[11] D. Brochier, Cryostat à température variable pour mesures neutroniques ou optiques, ILL Tech. Report 77/74, 1977.

[12] P. Fischer, G. Frey, M. Koch, M. Konnecke, V. Pomjakushin, J. Schefer, R. Thut, N. Schlumpf, R. Burge, U. Greuter, S. Bondt, and E. Berruyer, Phys. B: Condens. Matter 276-278, 146 (2000).

[13] J. K. Stalick, E. Prince, A. Santoro, I. G. Schroder, and J. J. Rush, MRS Proc. 376, 101 (1994).

[14] ${ }^{3} \mathrm{He}$ cryostat specifications available from https: //www.nist.gov/ncnr/sample-environment/equipment/liquidhelium-cryostats/3he-big-blue.

[15] Low- $T$ high-power CCR specifications are available from https://www.nist.gov/ncnr/sample-environment/equipment/ closed-cycle-refrigerators-ccr/low-t-high-power.

[16] J. Rodriguez-Carvajal, Solid State Phenom 170, 263 (2011).

[17] A. S. Wills, Z. Kristallogr. Suppl. 30, 39 (2009).

[18] K. Momma and F. Izumi, J. Appl. Crystallogr. 44, 1272 (2011).
[19] A. Amato, H. Luetkens, K. Sedlak, A. Stoykov, R. Scheuermann, M. Elender, A. Raselli, and D. Graf, Rev. Sci. Instrum. 88, 093301 (2017).

[20] GPS flow cryostat specifications available from https://www. psi.ch/en/smus/gps\#Flow_45Cryostat.

[21] P. Giannozzi, S. Baroni, N. Bonini, M. Calandra, R. Car, C. Cavazzoni, D. Ceresoli, G. L. Chiarotti, M. Cococcioni, I. Dabo, A. Dal Corso, S. De Gironcoli, S. Fabris, G. Fratesi, R. Gebauer, U. Gerstmann, C. Gougoussis, A. Kokalj, M. Lazzeri, L. Martin-Samos et al., J. Phys.: Condens. Matter 21, 395502 (2009).

[22] J. P. Perdew, K. Burke, and M. Ernzerhof, Phys. Rev. Lett. 77, 3865 (1996).

[23] J. Rodríguez-Carvajal, Fullprof Manual, available at https:// www.psi.ch/sites/default/files/import/sinq/dmc/ManualsEN/ fullprof.pdf.

[24] Q. Tao (unpublished).

[25] See Supplemental Material at http://link.aps.org/supplemental/ 10.1103/PhysRevB.104.174440 for additional data and symmetry analysis.

[26] E. F. Bertaut, Acta Crystallogr. Sect. A 24, 217 (1968).

[27] A. L. Patterson, Phys. Rev. 56, 978 (1939).

[28] A. Yaouanc and P. D. de Réotier, Muon Spin Rotation, Relaxation, and Resonance: Applications to Condensed Matter (Oxford University Press, Oxford, UK, 2011).

[29] A. Amato, R. Feyerherm, F. N. Gygax, A. Schenck, H. V. Löhneysen, and H. G. Schlager, Phys. Rev. B 52, 54 (1995).

[30] P. Bonfà, F. Sartori, and R. De Renzi, J. Phys. Chem. C 119, 4278 (2015).

[31] E. K. U. Gross and R. M. Dreizler, Density Functional Theory (Springer Science \& Business Media, New York, 2013).

[32] J. S. Smart, Effective Field Theories of Magnetism (Saunders, Philadelphia, PA, 1968).

[33] A. Auerbach, Interacting Electrons and Quantum Magnetism (Springer-Verlag, Berlin, 1994).

[34] B. Keimer, A. Aharony, A. Auerbach, R. J. Birgeneau, A. Cassanho, Y. Endoh, R. W. Erwin, M. A. Kastner, and G. Shirane, Phys. Rev. B 45, 7430 (1992).

[35] R. Ofer, G. Bazalitsky, A. Kanigel, A. Keren, A. Auerbach, J. S. Lord, and A. Amato, Phys. Rev. B 74, 220508(R) (2006).

[36] C. Lacroix, P. Mendels, and F. Mila, Introduction to Frustrated Magnetism: Materials, Experiments, Theory (Springer Science \& Business Media, New York, 2011).

[37] L. J. Chang, M. R. Lees, I. Watanabe, A. D. Hillier, Y. Yasui, and S. Onoda, Phys. Rev. B 89, 184416 (2014).

[38] T. Adachi, S. Yairi, K. Takahashi, Y. Koike, I. Watanabe, and K. Nagamine, Phys. Rev. B 69, 184507 (2004). 
[39] P. Dalmas De Réotier, A. Maisuradze, A. Yaouanc, B. Roessli, A. Amato, D. Andreica, and G. Lapertot, Phys. Rev. B 93, 144419 (2016).
[40] S. M. Disseler, Phys. Rev. B 89, 140413 (2014).

[41] P. G. De Gennes, J. Phys. Le Radium 23, 510 (1962).

[42] www.negevhpc.com. 